\title{
Influence of surface viscosity on droplets in shear flow
}

\author{
J. Gounley ${ }^{1}$, G. Boedec ${ }^{2}$, M. Jaeger ${ }^{1}$ and M. Leonetti ${ }^{2} \dagger$ \\ ${ }^{1}$ Aix-Marseille Université, CNRS, Centrale Marseille, M2P2 UMR 7340, Marseille 13451, France \\ ${ }^{2}$ Aix-Marseille Université, CNRS, Centrale Marseille, IRPHE UMR 7342, Marseille 13384, France
}

(Received 9 April 2015; revised 1 January 2016; accepted 9 January 2016)

The behaviour of a single droplet in an immiscible external fluid, submitted to shear flow is investigated using numerical simulations. The surface of the droplet is modelled by a Boussinesq-Scriven constitutive law involving the interfacial viscosities and a constant surface tension. A numerical method using Loop subdivision surfaces to represent droplet interface is introduced. This method couples boundary element method for fluid flows and finite element method to take into account the stresses due to the surface dilational and shear viscosities and surface tension. Validation of the numerical scheme with respect to previous analytic and computational work is provided, with particular attention to the viscosity contrast and the shear and dilational viscosities characterized both by a Boussinesq number $B_{q}$. Then, influence of equal surface viscosities on steady-state characteristics of a droplet in shear flow are studied, considering both small and large deformations and for a large range of bulk viscosity contrast. We find that small deformation analysis is surprisingly predictive at moderate and high surface viscosities. Equal surface viscosities decrease the Taylor deformation parameter and tank-treading angle and also strongly modify the dynamics of the droplet: when the Boussinesq number (surface viscosity) is large relative to the capillary number (surface tension), the droplet displays damped oscillations prior to steady-state tank-treading, reminiscent from the behaviour at large viscosity contrast. In the limit of infinite capillary number $\mathrm{Ca}$, such oscillations are permanent. The influence of surface viscosities on breakup is also investigated, and results show that the critical capillary number is increased. A diagram $\left(B_{q} ; \mathrm{Ca}\right)$ of breakup is established with the same inner and outer bulk viscosities. Additionally, the separate roles of shear and dilational surface viscosity are also elucidated, extending results from small deformation analysis. Indeed, shear (dilational) surface viscosity increases (decreases) the stability of drops to breakup under shear flow. The steady-state deformation (Taylor parameter) varies nonlinearly with each Boussinesq number or a linear combination of both Boussinesq numbers. Finally, the study shows that for certain combinations of shear and dilational viscosities, drop deformation for a given capillary number is the same as in the case of a clean surface while the inclination angle varies.

Key words: capsule/cell dynamics, complex fluids, drops and bubbles 


\section{Introduction}

Since the work of Taylor (1934), the case of droplets suspended in a simple, immiscible flow has received attention in experimental, theoretical and computational studies. Results from studies of droplet deformation have proved useful in informing investigations of other soft matter modelling paradigms, such as capsules or vesicles, which involve interfaces with more complicated mechanics (e.g. Stone 1994; Barthès-Biesel 2009; Vlahovska, Podgorski \& Misbah 2009b; Abreu et al. 2014). More directly, studies of droplets may be used to better understand the properties of emulsions providing an insight of rheological properties of interfaces. For instance, the critical state at which a droplet breaks apart helps to quantify emulsion stability (Fischer \& Erni 2007). Likewise, droplet studies may help to characterize the relationship between interfacial and hydrodynamic forces in emulsions.

In the simplest model, the interfacial stress between immiscible fluids may be described by a constant surface tension. However, the production of an emulsion or foam often involves the use of surfactants, which decrease the stress at the interface, and variations in surfactant concentration may lead to Marangoni flow (Pawar \& Stebe 1996; Erni 2011). Other contaminants in the fluids may provide additional interfacial stresses (Rumscheidt \& Mason 1961) and the interface may display the properties of a two-dimensional fluid (Boussinesq 1913). The properties of an interface characterised by surfactants and contaminants are necessary to understand the behaviour of emulsions and foams (Langevin 2000). As a result, a model of the interface must also account for these surface viscosities and gradients in surface tension.

Oldroyd (1955) derived a formula for the effective viscosity of a dilute emulsion with surface viscosity. Danov (2001) generalized Oldroyd's result to account for both surface viscosity and Marangoni stresses from surfactant concentration variations. However, the role of surface viscosity in other aspects of emulsion behaviour is somewhat unsettled. Of particular interest is the matter of how surface viscosity may be related to emulsion and foam stability and longevity. Georgieva et al. (2009) indicates that surface elasticity correlates well with emulsion stability with a related concern, Ostwald ripening. Experimental results have long indicated that shear surface viscosity is correlated with the stability of emulsion coalescence (Dickinson, Murray \& Stainsby 1988; Miller et al. 2010). However, first, Harvey et al. (2005) suggests that dilational surface viscosity should also be taken into account. Mun \& McClements (2006) measured a high dilational modulus of SDS-chitosan interface for example. Second, recent results by Zell et al. (2014) cast doubt on previous experimental measurements of shear surface viscosity and then on conclusions drawn from these measurements on emulsion stability. As briefly presented, the question of the measurement of surface viscosities is still under progress and debate. The respective roles of shear and dilational surface viscosities on interfacial dynamics also require clarification. In this paper, we choose to study numerically their roles on the shape of a droplet in shear flow.

Meanwhile, the computational and analytic work which followed Taylor began by considering a single 'clean' droplet, having an interface uncontaminated by impurities or surfactants, and therefore reasonably described by a constant surface tension. Cox (1969) used small deformation analysis to describe the time evolution of droplets in linear flows and accounted for the viscosity contrast between droplet and the external flow. The small deformation theory of Barthès-Biesel \& Acrivos (1973) explored the conditions for droplet breakup in shear flow. These conditions are quantified by the critical capillary number $C a_{c}$, beyond which a droplet does not have a stable steady 
state. Numerical work by Rallison \& Acrivos (1978) and Rallison (1981) studied the critical capillary number in shear and extensional flows and its dependence on the viscosity contrast. Kennedy, Pozrikidis \& Skalak (1994) used a boundary element method to estimate the critical viscosity contrast, which ensures a stable steady state and lets $C a_{c} \rightarrow \infty$. Subsequent numerical work (e.g. Li, Renardy \& Renardy 2000 and Cristini et al. 2003) has continued to clarify the relationship between the critical capillary number and viscosity contrast for clean droplets.

Numerical work has also explored how the presence and transport of surfactants alters droplet behaviour. Broadly speaking, surfactant transport on the surface encourages two competing processes, dilution and convection, which tend to decrease and increase droplet deformation, respectively. The effect of an insoluble surfactant on droplets with no viscosity contrast was studied by the boundary element methods of Stone \& Leal (1990) and Li \& Pozrikidis (1997), which showed that surfactant transport can either increase or decrease the critical capillary number. The numerical and experimental work of Feigl et al. (2007) showed that the impact of surfactants on the critical capillary number depended on the viscosity contrast. More comprehensive discussions of surfactants on droplets may be found in Feigl et al. (2007) and Fischer \& Erni (2007).

Analytical and numerical work has also considered droplets with Newtonian surface viscosity. The analysis of LeVan (1981) treated the Marangoni migration of droplets with surface viscosity in an external flow. Manor, Lavrenteva \& Nir (2008) extended LeVan's results to account for variable surface viscosities. Valkovska, Danov \& Ivanov (1999) studied how the deformation of bubbles, a related physical model, is affected by surface viscosity and Marangoni stress during collisions. More recently, the effect of surface viscosity on the dynamics of a spherical droplet in Poiseuille flow has been studied. Schwalbe et al. (2011) showed analytically how surface viscosity and Marangoni effects alter a droplet's migration velocity in Poiseuille flow and the velocity field inside the droplet. Subsequently, Reusken \& Zhang (2013) used a level-set model of two-phase flow to describe the droplet interface, and demonstrated convergence to the migration velocities for varied surface viscosities. Both Schwalbe et al. (2011) and Reusken \& Zhang (2013) used a Boussinesq-Scriven constitutive law to model the droplet surface.

However, few studies appear to have considered the deformation of a droplet with surface viscosity, as in a shear flow. The principal result is from Flumerfelt (1980), who extended the small deformation analysis of Cox (1969) for the deformation and inclination angle of droplets in linear flows to incorporate the additional effects of surface viscosity and small gradients in surface tension. Flumerfelt's results suggested that shear and dilational surface viscosity had rather distinct impacts on droplet deformation. Phillips, Graves \& Flumerfelt (1980) applied Flumerfelt's analysis to experimental results, finding that it held promise for measuring surface viscosity parameters of droplets.

Pozrikidis (1994) developed an early computational model of surface viscosity for spherical droplets in shear flow, generalizing the boundary element method presented in Kennedy et al. (1994) for clean droplet deformation. Pozrikidis' surface model was also based on a Boussinesq-Scriven constitutive law. However, results were limited by numerical stability and computational expense. As a result, steady-state values could not generally be computed and only droplets with equal shear and dilational surface viscosities were considered. Nonetheless, this work extended the existing theory from Flumerfelt's perturbation analysis, by considering larger deformations and suggesting that surface viscosity may increase the critical capillary number at 


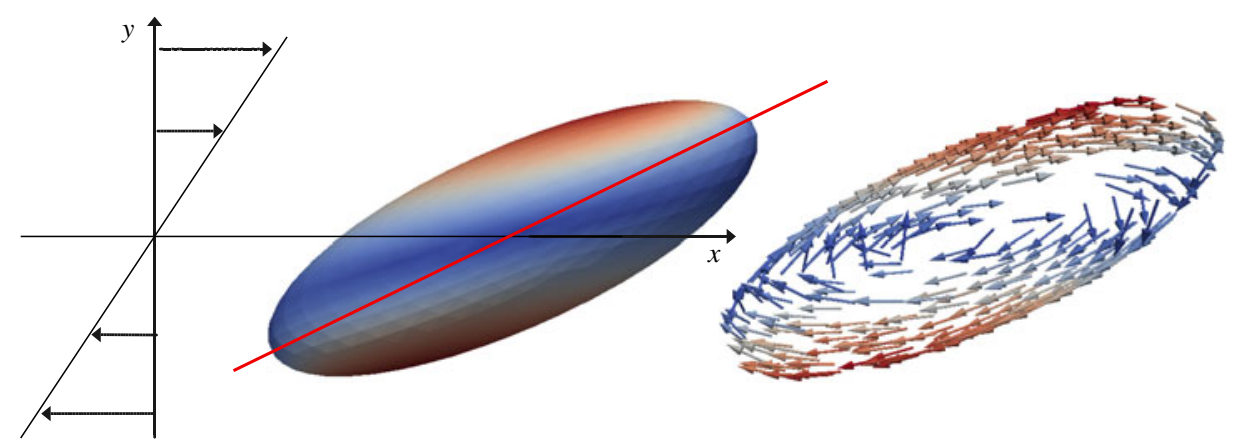

FIGURE 1. (Colour online) Deformation and inclination of a droplet in shear flow. The arrows indicate the flow pattern at the interface, its magnitude increasing from blue to red colour.

which a droplet breaks apart in flow. Interestingly, Yazdani \& Bagchi (2013) have studied the contribution of shear surface viscosity to the dynamics of capsules. They showed notably that the period of tank-treading varies with membrane viscosity, a result recently confirmed by experimental investigations on HSA microcapsules (de Loubens et al. 2015b). However, the computation of bending stiffness was found to be necessary for capsules, in order to suppress wrinkling. Moreover, the dilational surface viscosity has not been taken into account. The calculation of the dynamics of pearling instability along tubular vesicles Boedec, Jaeger \& Leonetti (2014) has been recently improved by taking into account the shear surface viscosity (Narsimhan, Spann \& Shaqfeh 2015). Here, the study is focused on droplets considering both the dilational and shear viscosities to better understand their respective contributions on shape dynamics of soft particles such as droplets.

In this work, we make a computational study of droplets with shear (figure 1) and dilational surface viscosity, immersed in an external flow and subject to both large and small deformations. Section 2.2 provides a physical description of the droplet surface and its relation to the external flow. For simplicity, surfactant transport and Marangoni stresses arising from gradients in surface tension are not considered. In this paper, one aim is to clearly understand the respective roles of shear and dilational viscosities. Section 2.3 implements this model numerically, with a Loop subdivision surface and finite element method. A boundary element model is introduced in $\S 2.4$, which describes the time evolution of the coupled drop-flow system and is implicit for droplet velocity. Validation of the numerical model is provided in $\S 3$, with respect to previous analytical and computational results considering the viscosity contrast and both surface viscosities. Section 4 contains results for the deformation and dynamics of droplets in shear flow, initially for identical shear and dilational surface viscosities and subsequently for purely shear or dilational surface viscosity. Results are compared with small deformation analysis and droplet dynamics during the approach to steady state are explored.

\section{Model}

\subsection{Fluid dynamics}

The physical system considered is a droplet with a viscous interface, centred in and surrounded by an abruptly started infinite Newtonian flow. In experiments on droplets 
and emulsions (Mun \& McClements 2006; Feigl et al. 2007), the characteristic length is droplet radius $a \sim 10-100 \mu \mathrm{m}$ and the characteristic velocity is $u_{0} \sim 100 \mu \mathrm{m} \mathrm{s}^{-1}$. In combination with density $\rho \sim 10^{3} \mathrm{~kg} \mathrm{~m}^{-3}$ and dynamic viscosity $\mu \sim 100 \mathrm{mPa}$, an approximate Reynolds number is not larger than $R e \sim 10^{-4}$ for previous experiments and reach $R e \sim 10^{-2}$ considering water. As a result, the dynamics of the fluid inside and outside of the drop are appropriately described by the Stokes equations

$$
-\nabla p+\mu \Delta v+f=0, \quad \nabla \cdot v=0
$$

for pressure $p$ and applied force $f$. In the following the inner and outer viscosities will be noted as $\mu^{i n t}$ and $\mu^{\text {ext }}$, respectively. Due to the linearity of the Stokes equations, the velocity $\boldsymbol{v}$ at the interface is the sum of the ambient flow $\boldsymbol{v}^{\infty}$ and a 'perturbation' resulting from the force density on the interface $\Gamma$. For shear flow, $\boldsymbol{v}^{\infty}=(\dot{\epsilon} y, 0,0)$, for shear rate $\dot{\epsilon}$ (figure 1). For Poiseuille flow, $\boldsymbol{v}^{\infty}$ is described later in (3.6). Far from the drop interface, as $|\boldsymbol{x}| \rightarrow \infty$, the influence of the interfacial forces vanishes and the boundary conditions are

$$
\boldsymbol{v} \rightarrow \boldsymbol{v}^{\infty} \text { and } \quad p \rightarrow 0
$$

The droplet and ambient fluid are assumed to be immiscible, but their dynamics are coupled. Velocity is continuous across the droplet surface $\Gamma$. Thus, at point $\boldsymbol{x}$ on $\Gamma$,

$$
\boldsymbol{v}^{e x t}(x)=\boldsymbol{v}^{i n t}(x)=\boldsymbol{v}^{s}(x)
$$

for external and internal fluid velocities $\boldsymbol{v}^{\text {ext }}$ and $\boldsymbol{v}^{\text {int }}$. As a result of the immiscibility, fluid does not flow across the droplet surface and the droplet volume does not change. As a result, the fluid velocities are identical to the velocity of the membrane,

$$
\frac{\mathrm{D} x}{\mathrm{D} t}=\boldsymbol{v}^{s}(\boldsymbol{x})
$$

for $\boldsymbol{x}$ on $\Gamma$ and material derivative $\mathrm{D} / \mathrm{D} t$. Additionally, the droplet surface is assumed to be at mechanical equilibrium with hydrodynamic forces provided by the hydrodynamic stress tensor $\bar{\sigma}$. This quasistatic equilibrium requires a surface force density $f$, as

$$
[[\overline{\boldsymbol{\sigma}}]] \cdot \boldsymbol{n}+\boldsymbol{f}=0
$$

in which $[[\overline{\boldsymbol{\sigma}}]]=\overline{\boldsymbol{\sigma}}^{\text {ext }}-\overline{\boldsymbol{\sigma}}^{\text {int }}$ is the traction jump at the droplet surface $\Gamma . \boldsymbol{n}$ is the unit outward normal vector to the surface.

\subsection{Mechanical properties of the surface}

The mechanical properties of the droplet surface are represented by the BoussinesqScriven stress tensor, originally described by Boussinesq (1913) and generalized by Scriven (1960). A Boussinesq-Scriven surface is characterized by a linear constitutive law with surface tension $\gamma$, shear surface viscosity $\mu_{s}$ and dilational surface viscosity $\mu_{d}$. In these terms, the surface stress tensor $\overline{\boldsymbol{\sigma}}_{s}$ may be formulated as

$$
\overline{\boldsymbol{\sigma}}_{s}=\gamma \overline{\boldsymbol{P}}+\left(\mu_{d}-\mu_{s}\right) \Theta \overline{\boldsymbol{P}}+2 \mu_{s} \overline{\boldsymbol{e}}
$$

for surface projection tensor $\overline{\boldsymbol{P}}$, surface rate of dilation $\Theta$ and surface rate of deformation tensor $\overline{\boldsymbol{e}}$ (Secomb \& Skalak 1982). $\overline{\boldsymbol{P}}$ is defined using the unit outward 
normal vector $\boldsymbol{n}$ to the surface and the unit tensor $\overline{\boldsymbol{I}}$, as

$$
\overline{\boldsymbol{P}}=\overline{\boldsymbol{I}}-\boldsymbol{n} \boldsymbol{n}^{\mathrm{T}} .
$$

In terms of the surface gradient $\nabla_{s}$ and surface velocity $\boldsymbol{v}^{s}$, the surface rate of deformation tensor $\overline{\boldsymbol{e}}$ is given by

$$
\overline{\boldsymbol{e}}=\frac{1}{2} \overline{\boldsymbol{P}}\left(\boldsymbol{\nabla}_{s} \boldsymbol{v}^{s}+\left(\boldsymbol{\nabla}_{s} \boldsymbol{v}^{s}\right)^{\mathrm{T}}\right) \overline{\boldsymbol{P}}
$$

and the surface rate of dilation $\Theta$ by

$$
\Theta=\overline{\boldsymbol{P}}: \nabla_{s} \boldsymbol{v}^{s} .
$$

In this paper, both shear and dilational surface viscosities $\mu_{s}$ and $\mu_{d}$ are constant. Likewise, surface tension $\gamma$ is also constant and, in the absence of an external flow, the droplet's equilibrium shape is spherical. As a result, droplets in subsequent simulations are initially spherical.

\subsection{Numerical model of the surface}

The numerical model represents the droplet interface with Loop elements as a subdivision surface (Loop 1987; Cirak, Ortiz \& Schroder 2000), a powerful method to computer aided design. Using a triangular mesh based on successive refinements of an icosahedron, the subdivision method guarantees $C^{2}$ continuity almost everywhere on the surface (except on the 12 vertices of the initial icosahedron, where the surface is only $C^{1}$ ). However, these vertices do not necessitate special treatment, since membrane forces are computed by a finite element method which relaxes the continuity requirement compared to a direct computation (see below for more details). The characteristic feature of a subdivision surface is that the displacement field of an element depends on the nodal displacement of nearest-neighbour elements, in addition to its own nodal displacement. These nearest-neighbour elements are said to comprise the 1-ring about the element; regular elements have 12 elements in their 1-ring. For a given element $e$, position $\boldsymbol{x}$ is computed as

$$
\boldsymbol{x}(\xi, \eta)=\sum_{n \in E_{n}} N_{n}^{e}(\xi, \eta) \boldsymbol{x}_{n}
$$

for the curvilinear coordinates $(\xi, \eta)$ on element $e$, node $n$ in the 1-ring $E_{n}$ about element $e$, box-spline shape functions $N_{n}^{e}$ and the nodal value $\boldsymbol{x}_{n}$. The velocity $\boldsymbol{v}(\xi, \eta)$ on the surface is computed in the same way as $\boldsymbol{x}$. The box-spline shape functions used for both regular and irregular elements are discussed in the appendix of Cirak et al. (2000). This surface representation provides accurate derivatives with respect to $\xi$ and $\eta$, which are necessary to compute the surface stress tensor. This method provides an improvement compared to our previous code and notably for the surface representation (Boedec, Leonetti \& Jaeger 2011; Boedec, Jaeger \& Leonetti 2012). Two examples of meshing of droplets with dilational viscosity in shear flow for two numbers of elements are shown in figure 2. Loop subdivision elements have been used in previous studies on capsules (Le 2010; Huang et al. 2012) and vesicles (Spann, Zhao \& Shaqfeh 2014). However, the numerical methods to compute membrane stress and the fluid solver are different. 

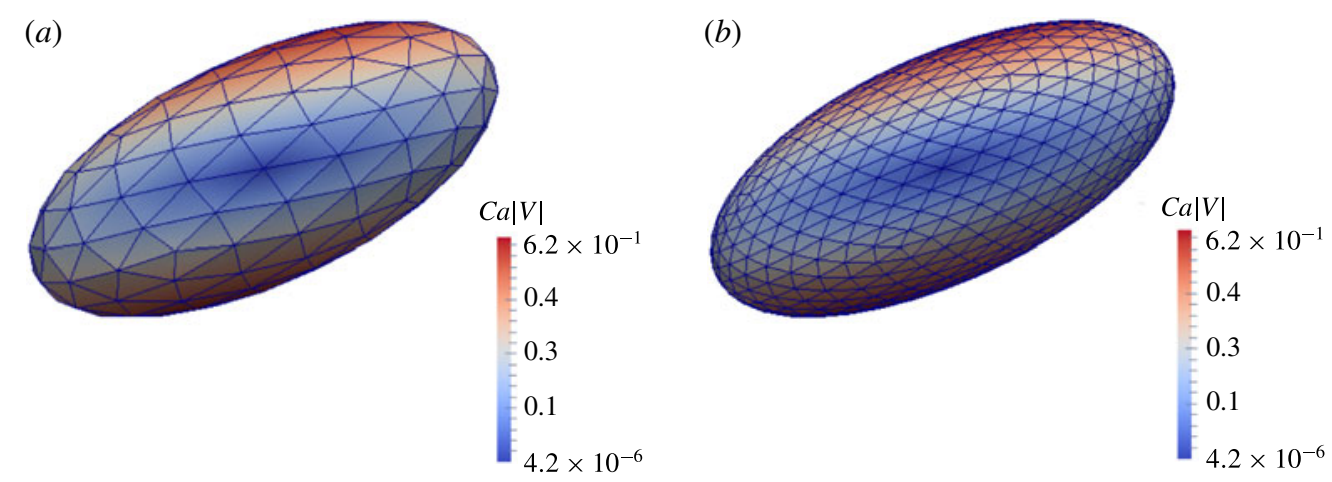

Figure 2. (Colour online) Two cases of meshing characterized by the number $N$ of elements of a capsule with dilational viscosity in shear flow: $C a=0.3, B q_{s}=0$ and $B q_{d}=1$. The colour code corresponds to the magnitude of the interface velocity. (a) $N=320,(b)$ $N=1280$.

With this framework established, the surface velocity gradient is computed on each element as

$$
\nabla_{s} \boldsymbol{v}=a^{\alpha \beta} \frac{\partial v_{i}}{\partial s^{\alpha}} \frac{\partial x_{j}}{\partial s^{\beta}} e_{i} \otimes e_{j},
$$

with Cartesian components of velocity $v_{i}$ and position $x_{j}$, local curvilinear coordinates $s^{\alpha}=(\xi, \eta)$ and the local contravariant metric tensor $a^{\alpha \beta}$. With (2.11) substituted into the components of (2.6), the Boussinesq-Scriven stress tensor may be computed. Transformed to curvilinear coordinates, the stress tensor takes the form

$$
\sigma^{\alpha \beta}=a^{\alpha \gamma} a^{\beta \delta} \frac{\partial x_{i}}{\partial s^{\gamma}} \frac{\partial x_{j}}{\partial s^{\delta}} \sigma_{i j} .
$$

We follow the finite element method described by Cirak et al. (2000) to solve the weak form of the equation

$$
\nabla_{s} \cdot \sigma^{\alpha \beta}-\boldsymbol{f}=0
$$

for surface force density $\boldsymbol{f}$. Introducing a virtual displacement field $\delta \boldsymbol{x}$, (2.13) reads:

$$
\int_{S}\left(\frac{1}{2} \sigma^{\alpha \beta} \delta a_{\alpha \beta}+\boldsymbol{f} \cdot \delta \boldsymbol{x}\right) \mathrm{d} S=0, \quad \forall \delta \boldsymbol{x},
$$

where $\delta a_{\alpha \beta}=\delta \boldsymbol{x}_{, \alpha} \cdot \boldsymbol{x}_{, \beta}+\boldsymbol{x}_{, \alpha} \cdot \delta \boldsymbol{x}_{, \beta}$ is the virtual variation of the metric. Thus, with the weak form of membrane equilibrium, only first derivatives of shape and virtual displacement are needed to compute membrane forces. Using the same interpolation basis (Loop elements) for the virtual displacement $\delta \boldsymbol{x}$ and for the force $\boldsymbol{f}$, this equation writes:

$$
\sum_{e=1}^{N_{e l}} \int_{S^{e}} \sum_{n=1}^{N^{e}} \sum_{m=1}^{N^{e}}\left(\frac{1}{2} \sigma^{\alpha \beta}\left[\left(N_{n, \alpha} N_{m, \beta}+N_{n, \beta} N_{m, \alpha}\right) \delta x_{i}^{n} x_{i}^{m}\right]+N_{n} N_{m} f_{i}^{n} \delta x_{i}^{m}\right) \mathrm{d} S^{e}=0,
$$

where the integral over the surface $S$ has been split as a sum of integration over element $e$ surface $S^{e}$, with $N_{e}$ elements in the mesh and the interpolation (2.10) by Loop element has been used, with $N_{n}$ as a notation for the $n$th shape function of 
element $e$, evaluated at coordinates $(\xi, \eta): N_{n}=N_{n}^{e}(\xi, \eta)$ and $x_{i}^{n}$ stands for the nodal value $n$ of the $i$ th Cartesian component of position. The integration over surfaces $S^{e}$ is computed using Gauss quadrature points. Thus, after rearranging the terms, and solving the linear system with the nodal values of Cartesian component of force $f_{i}^{n}$, the computation of $\boldsymbol{f}$ may be then formulated symbolically in terms of linear operators $F^{\gamma}$ and $F^{\nu}$ acting on $\gamma$ and $\boldsymbol{v}$, respectively, as

$$
\boldsymbol{f}=F^{\gamma}(\gamma)+F^{v}(\boldsymbol{v})
$$

\subsection{Boundary element model and update scheme}

The Stokes equations are solved using the standard boundary element method (Pozrikidis 1992). The boundary element method is evaluated over the same mesh used to describe the droplet surface and velocity is assumed to be continuous across this interface. For Cartesian component $i$ of the velocity $\boldsymbol{v}$ at $\boldsymbol{x}$, the boundary integral is

$$
\begin{aligned}
v_{i}(\boldsymbol{x})= & v_{i}^{\infty}(\boldsymbol{x})+\frac{1}{8 \pi \mu^{e x t}} \int_{S} G_{i j}\left(\boldsymbol{x}_{0}, \boldsymbol{x}\right) f_{j}\left(\boldsymbol{x}_{0}\right) \mathrm{d} S\left(\boldsymbol{x}_{0}\right) \\
& +\frac{1-\lambda}{8 \pi} \int_{S}\left[v_{i}\left(\boldsymbol{x}_{\mathbf{0}}\right)-v_{i}(\boldsymbol{x})\right] T_{i j k}\left(\boldsymbol{x}, \boldsymbol{x}_{\mathbf{0}}\right) n_{k}\left(\boldsymbol{x}_{\mathbf{0}}\right) \mathrm{d} S\left(\boldsymbol{x}_{\mathbf{0}}\right),
\end{aligned}
$$

with free space Stokeslet $G_{i j}=\left(\delta_{i j} / r\right)+\left(X_{i} X_{j} / r^{3}\right)$ and Stresslet $T_{i j k}=-6\left(X_{i} X_{j} X_{k} / r^{5}\right)$, point $\boldsymbol{x}_{0}$ on $\Gamma$, vector $\boldsymbol{X}=\boldsymbol{x}_{0}-\boldsymbol{x}$ and $r=\|\boldsymbol{X}\|$. The viscosity contrast has been introduced as follows:

$$
\lambda=\mu^{i n t} / \mu^{e x t} .
$$

As a result, at point $\boldsymbol{x}$ on $\Gamma$, the discretized version of (2.17) may be written as

$$
\boldsymbol{v}=\boldsymbol{v}^{\infty}+\boldsymbol{G} \boldsymbol{f}+(1-\lambda) \boldsymbol{T} \boldsymbol{v} .
$$

Taking into account the representation of the interfacial load in (2.16), the velocity of the droplet interface $v(t)$ is computed by solving the equation

$$
\boldsymbol{v}(\boldsymbol{x})=\boldsymbol{v}^{\infty}(\boldsymbol{x})+\boldsymbol{G}\left(F^{\gamma}(\gamma)+F^{v}(\boldsymbol{v})\right)+(1-\lambda) \boldsymbol{T} \boldsymbol{v} .
$$

This equation could be integrated explicitly in time, using the previous velocity field $\boldsymbol{v}^{t}$ to compute $\boldsymbol{x}^{t+\mathrm{d} t}$. Doing so would result in a constraint on the time step depending both on surface tension and surface viscosities. To avoid the constraint due to surface viscosity, we choose instead to solve (2.20) at each substep of the time stepping algorithm. Thus, as the equation is implicit for velocity $\boldsymbol{v}(t)$, it is recast as

$$
\left(I-\boldsymbol{G} F^{v}-(1-\lambda) \boldsymbol{T}\right) \boldsymbol{v}(\boldsymbol{x})=\boldsymbol{v}^{\infty}(\boldsymbol{x})+\boldsymbol{G} F^{\gamma}(\gamma) .
$$

A generalized minimum residual (GMRES) solver is used to solve (2.21) at each time step, providing a fully implicit solution for $\boldsymbol{v}(t)$. As a result, this approach differs from similar methods employing Boussinesq-Scriven stress, such as Rodrigues et al. (2015), which provide only semi-implicit solutions to the fully coupled problem. The cost of an implicit solution and iterative method, of course, is that multiple linear systems of equations are solved at each time step. Nonetheless, the iterative approach ensures that the primary restriction on time step $\mathrm{d} t$ is surface tension, not surface viscosity. According to the no-slip boundary condition in (2.4), updated position $\boldsymbol{x}(t+\mathrm{d} t)$ on the 
droplet is computed using the Runge-Kutta-Fehlberg method RK45, with an adaptive step size $\mathrm{d} t$. The same method has been recently used to calculate the dynamics of an elastic capsule without bending resistance in a planar elongation flow (de Loubens et al. 2015a).

As the droplet elongates in flow, the quality of the elements composing the mesh might degrade, especially for near-breakup simulations. Note that the inclusion of surface viscosities tends generally to smooth the elongation of elements on the whole mesh, thus leading to a better overall mesh quality. When needed, we use a remeshing algorithm which updates the position of the nodes while preserving the deformed shape. To do so, we slide the nodes tangentially on the surface, in order to have more nodes in regions of high curvature, while also keeping area of elements locally homogeneous.

\subsection{Dimensionless parameters and variables}

Several dimensionless parameters describe the surface tension and viscosities. The ratio of hydrodynamic stress to the resistance of surface tension $\gamma$ is reflected in the capillary number (or Weber number)

$$
C a=\frac{\mu^{e x t} \dot{\epsilon} a}{\gamma}
$$

for ambient fluid viscosity $\mu^{\text {ext }}$, shear rate $\dot{\epsilon}$ and initial droplet radius $a$. Borrowing the terminology of Erni (2011), surface viscosity strengths are measured by the dimensionless Boussinesq numbers,

$$
\begin{aligned}
& B q_{s}=\frac{\mu_{s}}{\mu^{e x t} a} \\
& B q_{d}=\frac{\mu_{d}}{\mu^{e x t} a}
\end{aligned}
$$

characterizing shear and dilational surface viscosity, respectively. When $B q_{s}=B q_{d}$, this single quantity is abbreviated by the Boussinesq number $B q$. A droplet with $B q=0$ is said to be 'clean'. Finally, $\beta$ describes the relative magnitudes of surface viscosity and tension as

$$
\beta=(B q)(C a) .
$$

Introduced by Barthès-Biesel \& Sgaier (1985) for capsules with surface viscosity, $\beta$ is comparable to the Weissenberg number in rheology. In the part on the results of this paper, we discuss clean drops with a contrast of viscosity $\lambda$ and drops with a viscous interface.

Additionally, physical quantities are non-dimensionalized: the lengths by the droplet radius a, the time by the inverse of the shear rate $1 / \dot{\epsilon}$, the pressure and membrane stress by $\mu^{e x t} \dot{\epsilon}$.

\section{Validation}

The numerical model in general, and the surface viscosity model in particular, are validated for droplets in shear and Poiseuille flows. In both settings, numerical convergence and good agreement with analytic or computational results are demonstrated in a large range of viscosity contrast and capillary number. Notably, the comparison of the inclination angle in the small capillary number limit establishes clearly this consistency. 
3.1. Clean droplet in shear flow

The shape of a clean droplet $(B q=0)$ in shear flow has been extensively studied numerically in the past using the boundary element method (Rallison 1981; Kennedy et al. 1994), advancing front method (Kwak \& Pozrikidis 1998) and volume-of-fluid method (Li et al. 2000), among many others. Such computational work has been shown to be consistent with experimental results (Rumscheidt \& Mason 1961; Kwak \& Pozrikidis 1998). Many authors have also determined well known analytical results based on the main assumption of small deformation theory: (Taylor 1934; Chaffey \& Brenner 1967; Cox 1969; Barthès-Biesel \& Acrivos 1973; Rallison 1980; Vlahovska, Blawzdziewicz \& Loewenberg 2005, 2009a). These approaches have their own domain of validity (Acrivos 1983; Rallison 1984). We recall below the analytical results well discussed in the literature which are sufficient for the validation of the code.

The shape in shear flow is mainly characterized by the so-called Taylor deformation parameter $D=(L-B) /(L+B)$, in which $L$ and $B$ are the major and minor axes of the ellipsoid having the same moment of inertia as the droplet. These axes remain in the $z=0$ plane for the duration of the simulations. Another salient parameter is the inclination $\theta$ which represents the angle between the direction of flow and the major axis of the droplet.

In his seminal work, Taylor (1934) has determined $D$ at the first order in $\mathrm{Ca}$ and inclination $\theta$ at the zeroth order in $\mathrm{Ca}$ in both limits of low and high viscosity contrast $\lambda$ :

$$
\begin{gathered}
\lambda \gg 1 ; \quad C a=O(1) \rightarrow D_{T}=\frac{5}{4 \lambda} ; \quad \theta=0 \\
\lambda=O(1) ; \quad C a \ll 1 \rightarrow D_{T}=C a \frac{19 \lambda+16}{16 \lambda+16} ; \quad \theta=\frac{\pi}{4} .
\end{gathered}
$$

Cox (1969) has proposed compact analytical equations which cover a large range of viscosity contrast assuming slight deformations from the spherical shape which can be quantified for example by $D \ll 1$ :

$$
D_{C o x}=\frac{C a \frac{19 \lambda+16}{16 \lambda+16}}{\sqrt{1+\left(\frac{19 \lambda C a}{20}\right)^{2}}} ; \quad \theta_{C o x}=\frac{\pi}{4}-\frac{1}{2} \operatorname{atan}\left(\frac{19 \lambda C a}{20}\right) .
$$

If the viscous stress is large $\lambda C a \gg 1$ (or very small), (3.1) (or (3.2)) is obtained. Further analysis has shown that the equations of Cox (1969) are in fact valid in the limit of large viscosity contrast (Rallison 1980). However, these equations are useful to validate our code, to highlight the global correct agreement between theory and numerics, the consistence of the high viscosity contrast limit and the need of high numerical accuracy to distinguish numerically models even if theory has already established their respective validities.

We performed numerical simulations of a clean droplet in shear flow covering 12 decades of viscosity contrast from $10^{-6}$ to $10^{6}$. The capillary number was chosen as $\mathrm{Ca}=10^{-3}$ to be consistent with the assumption of small deformation of models. The number of elements were 5120 elements without remeshing and 1280 elements with remeshing without notable differences. Both limits of Taylor (1934) are recovered as shown in figures 3 for the deformation and 4 for the inclination angle 


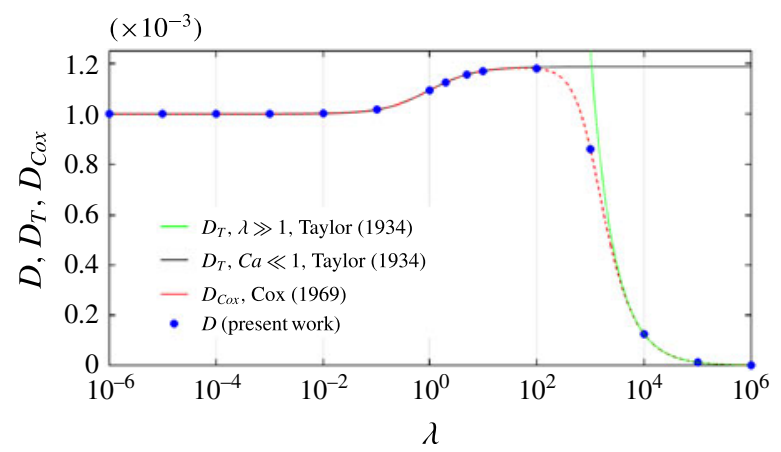

Figure 3. (Colour online) Clean droplet. Steady-state deformation: the Taylor parameter $D=(L-B) /(L+B)$ of a clean droplet in the limit of small capillary number $C a=10^{-3}$. Comparisons are made with analytical results of Taylor (1934) and Cox (1969).

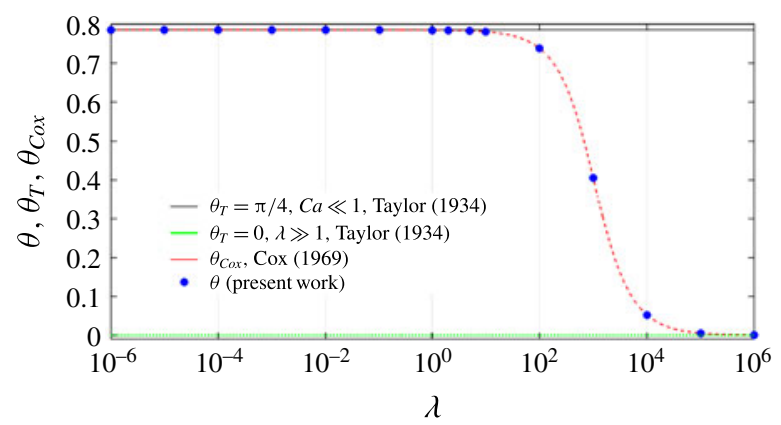

FIguRE 4. (Colour online) Clean droplet. Steady-state inclination angle $\theta$ of a clean droplet in the limit of small capillary number $C a=10^{-3}$. Comparisons are made with analytical results of Taylor (1934) and Cox (1969).

at weak and high viscosity contrasts. In the case of the Taylor parameter, the error $\left|D-D_{C o x}\right| / D_{C o x}$ on the Taylor parameter $D$ is approximately $3.0 \times 10^{-5}$ for $\lambda<10$, less than $3.0 \times 10^{-6}$ for $\lambda \geqslant 10^{5}$ and reaches the maximum $2.0 \times 10^{-4}$ for $\lambda=100$. This very good agreement could be due to the choice of a very small capillary number which is true in part. Then, the capillary number has also been varied from 0.001 to 0.4 for a range of viscosity contrast from 2 to 100 : figure 5 . We have checked that the slopes of numerical simulations of $D$ versus the capillary number are in excellent agreement with the theory of Taylor (1934). It is quite surprising that the results are in good agreement with the equations of Cox (1969) up to $C a=0.1$ for a large range of viscosity contrast. But they become not satisfactory for $C a \geqslant 0.3$. To conclude on the Taylor parameter of deformation $D$, the comparisons between numerics, analytical results of Taylor (1934) and Cox (1969) are very satisfactory at high viscosity contrast as expected but also at small capillary numbers. However, the Cox (1969) model should fail to describe accurately the shapes of droplets at moderate and small capillary numbers (Rallison 1980). Our numerical code should be able to highlight this discrepancy on the linear variations of deformation parameters and provides additional information on the domains of validities of models.

At $C a=10^{-3}$, the error on the inclination angle is larger, of the order of $10^{-3}$. Thus, we can expect a larger error at higher capillary numbers such as 0.1 for example 


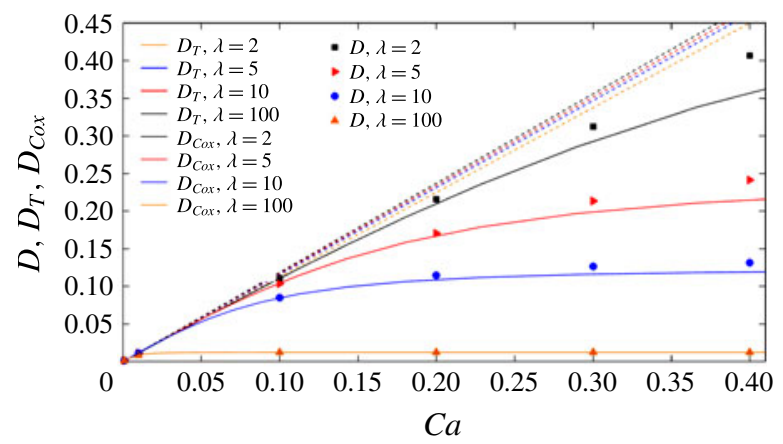

FigurE 5. (Colour online) Clean droplet. Steady-state deformation for a large range of capillary numbers $\mathrm{Ca}$ from 0.001 to 0.4 and for various viscosity contrasts $\lambda$ equal to 2, 5, 10 and 100.

which is not the case for $D$. In the limit of small capillary number and small deformation, Chaffey \& Brenner (1967) derived another equation for the inclination angle as a function of viscosity contrast $\lambda$ and capillary number $\mathrm{Ca}$ :

$$
\theta_{C B}=\frac{\pi}{4}-C a \frac{(19 \lambda+16)(2 \lambda+3)}{80(1+\lambda)} .
$$

This equation has been recovered by several authors (Barthès-Biesel \& Acrivos 1973; Rallison 1980; Vlahovska et al. 2005, 2009a) who calculate the power expansion in a small parameter, mainly the capillary number $\mathrm{Ca}$, of all the physical quantities: shape deformation, curvature, pressure, velocities and stress tensor. It means in particular that the viscous stress must be small, $\lambda C a \ll 1$ contrary to the model of Cox (1969). Note that this result has also been validated experimentally (Guido \& Villone 1998; Guido, Greco \& Villone 1999).

This relation is completely different from (3.3). Indeed, for a zero viscosity contrast, the result of Cox (1969) is $\pi / 4$ whatever the capillary number in agreement with Taylor (1934) but in contradiction with the expression of Chaffey \& Brenner (1967): $\theta_{C B}=\pi / 4-(3 / 5) C a$. Moreover, it is possible to develop (3.3) in the limit of small viscous stress $\lambda C a \ll 1$ providing the linear variation of the inclination with the capillary number, highlighting the difference between the theoretical results. Thus, $\theta_{C o x}=\pi / 4-C a(19 \lambda / 40)$. This is especially important to validate our numerical code. With a fixed viscosity contrast, the slopes are different meaning that the code must be able to differentiate clearly the models by a careful measurement of the inclination angle. Moreover, a linear variation as (3.4) valid for small viscous stress and $\mathrm{Ca}$ is always an opportunity to validate a numerical code. It is sufficient to decrease the capillary number up to a clear linear variation and to measure the slope. To perform this, we have varied the capillary number from 0.001 to 0.4 and the viscosity contrast from 1 to 100 . The number of elements was 1280 or 5120. The residual of GMRES was fixed to $10^{-9}$ for the resolution of (2.21) and the precision to $10^{-8}$ for the selection of the maximal step size in the RK45 time integrator. As expected, for $\lambda C a>1$, agreement is not reached with the (3.4) but there is good agreement with the Cox results as shown in figure 6(a). On the contrary, for $\lambda C a<1$, the agreement is excellent with (3.4): figure $6(b)$ and the curve $\lambda=1$ for figure $6(a)$. If this comparison allows us to validate our code for a clean droplet in Stokes flow, our calculations also 

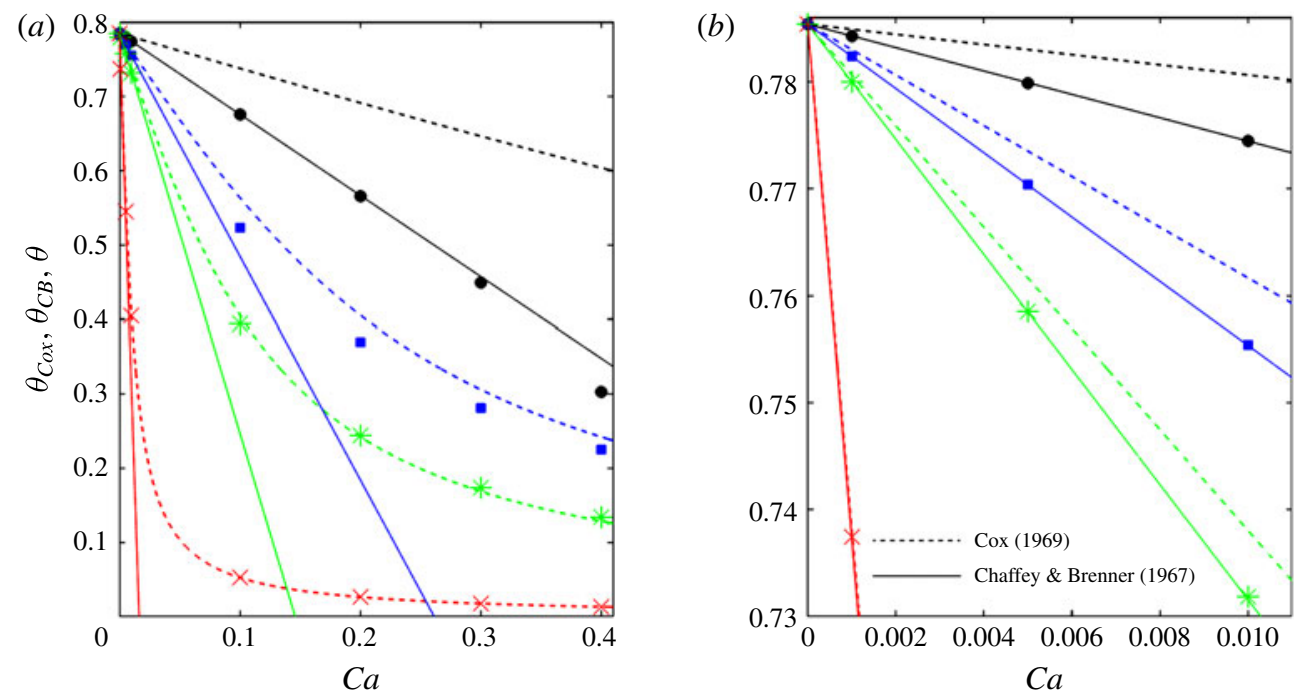

FIgURE 6. (Colour online) Clean droplet. Inclination angle as a function of the capillary number $\mathrm{Ca}$ and the viscosity contrast: dashed lines (full Cox equation), line (linear Chaffey-Brenner equation), symbols (numerical simulations). Colour code corresponds to $\lambda=1$ (black), $\lambda=5$ (blue), $\lambda=10$ (green) and $\lambda=100$ (red, $x$ ). Symbol code corresponds to $\lambda=1$ (disc), $\lambda=5$ (square), $\lambda=10$ (asterisk) and $\lambda=100$ (cross).

permit us to extract the limit of validity of (3.4) which is $\lambda C a \leqslant 0.2$. Indeed, with this criterion and regardless of the viscosity, the agreement between the result of Chaffey \& Brenner (1967) and our numerical results are excellent. Numerical studies on drops and capsules focus often on the Taylor parameter of deformation $D$, and more scarcely on the inclination angle while in this study, the latter is however essential to validate our code by differentiating unambiguously the linear models. Indeed, without deformation, the droplet radius is known while the inclination angle is not definite at this zeroth order. When the droplet deforms slightly from the spherical shape, the first order corresponds to the equations of Taylor (1934) which provides an inclination angle of $\pi / 4$ and a linear variation of $D$ with $C a$. Thus, equation of Chaffey \& Brenner (1967) provides an upper order of the inclination angle which is more dependent on models while remaining linear.

In the literature, to our knowledge, the case $\lambda=1$ has mainly been studied for comparisons with analytical expressions and to validate numerical codes: figure 7. Our results depart from linear theory earlier than Kwak \& Pozrikidis (1998), at $C a=0.25$, but match very well Li et al. (2000), in doing so considering the Taylor parameter $D$. The inclination angle $\theta$ differs more among the numerical studies in the literature. The results of Kennedy et al. (1994) and $\mathrm{Li}$ et al. (2000) do not really match the linear theory of Chaffey \& Brenner (1967) but provide a good approximation and in any case, are largely better than the linear result of Cox (1969). Indeed, the slopes at $\lambda=1$ are $35 / 32 \approx 1.09$ (Chaffey \& Brenner 1967) compared with $0.5 *(19 / 20)=0.475$ (Cox 1969). While the previous numerical results are performed for a capillary number larger than 0.1 , the present work extends the study to a minimum capillary number of $10^{-3}$, allowing a full comparison with linear theory, notably in the more difficult case of an inclination angle. An additional concern for this line of inquiry is the critical capillary number $C a_{c}$, beyond which a steady-state deformation does not exist for a 

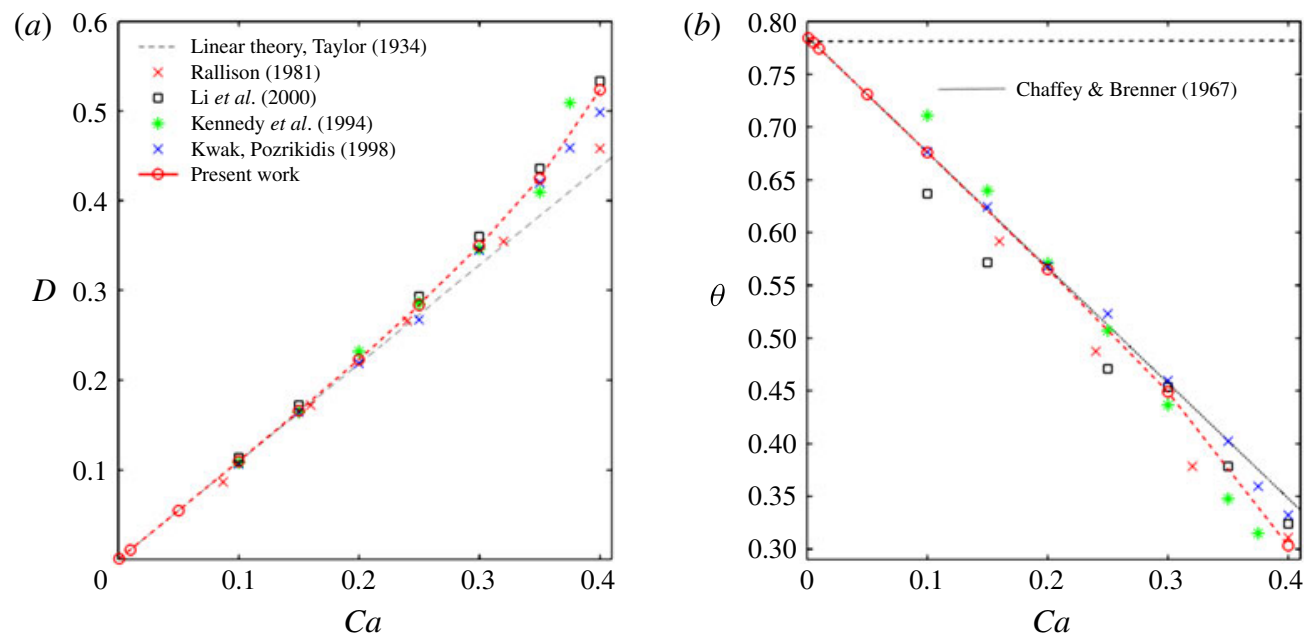

FIgURE 7. (Colour online) Clean droplet with $\lambda=1$. Comparisons with previous numerical studies. $(a)$ The Taylor parameter $D$ versus the capillary number $C a$. $(b)$ The inclination angle versus $\mathrm{Ca}$.

clean droplet with $\lambda=1$. We find the critical value $C a_{c} \approx 0.43$, which is consistent with the range $0.37-0.43$ found in previous computational and experimental studies (Rallison 1981; Kennedy et al. 1994; Li et al. 2000; Cristini et al. 2003; Fischer \& Erni 2007).

\subsection{Droplet with viscous interface in shear flow}

First, to evaluate the accuracy and convergence of the surface viscosity model, we consider a spherical droplet with $C a=\infty$ and $B q=B q_{s}=B q_{d}=1$ placed in shear flow. The initial viscous force distribution on the surface is computed and compared with the analytical values for each mesh. If the velocity far from the droplet is $\boldsymbol{V}=\dot{\epsilon} y e_{x}$, the analytical viscous force is $f=\left(-3 y+8 y x^{2}\right) e_{x}+\left(-3 x+8 x y^{2}\right) e_{y}+8 x y z e_{z}$. Error is computed by calculating the $\ell^{2}$ norm of the relative error at each node and using the Voronoi region about each node to integrate over the surface of the sphere. As seen in figure $8(a)$, first-order convergence of the error with respect to the number of elements $N$ is observed.

Second, to evaluate the numerical convergence over the duration of a simulation, we consider a droplet with a viscous surface in shear flow. The dependence of the deformation $D$ on grid refinement (or number of elements) is shown in figure $8(b)$, for two droplets with different viscosity contrast and Boussinesq number in shear flow. While there is noticeable error for the coarsest mesh (using only 80 elements), finer meshes agree very well for both sets of parameters. Subsequent simulations are conducted with 320 elements except for the breakup which can necessitate 1280 elements with remeshing or more.

\subsection{Droplet with viscous interface in Poiseuille flow}

A third validation setting of a viscous interface is provided by Poiseuille flow. Recent studies of droplets with viscous surfaces, Schwalbe et al. (2011) and Reusken \& Zhang (2013), consider the migration velocity of a droplet in Poiseuille flow and 

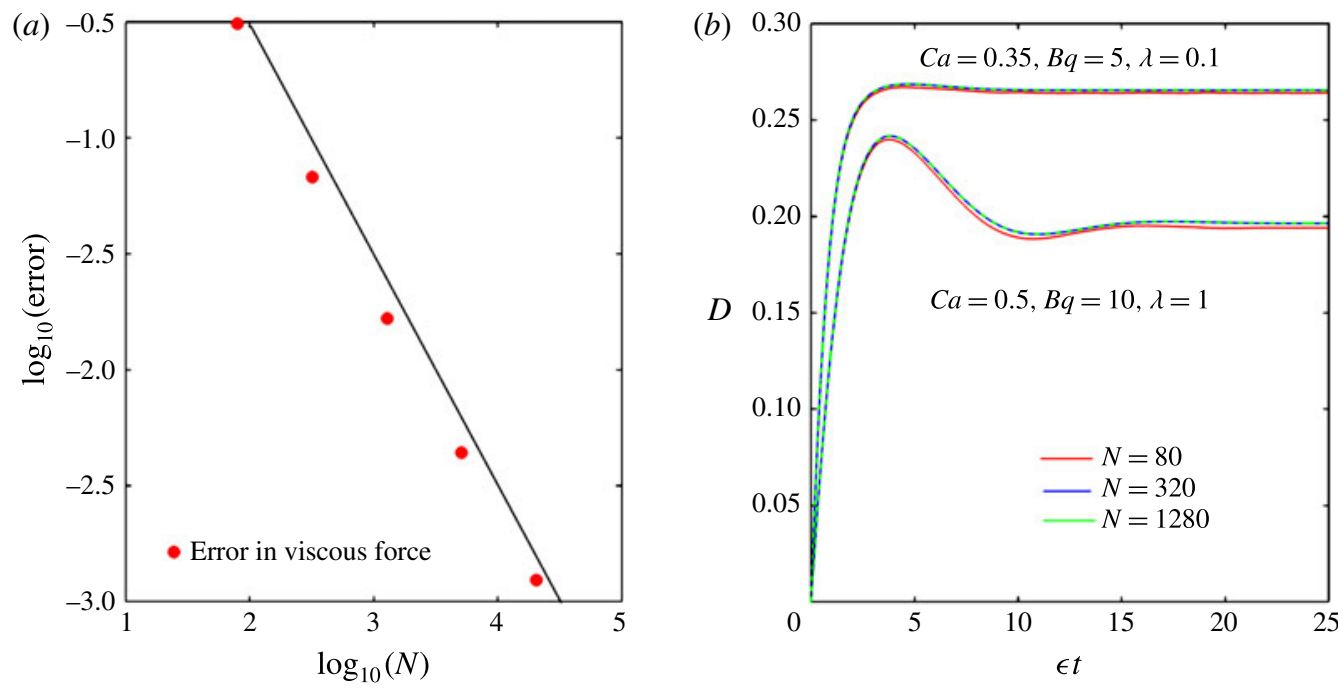

FIGURE 8. (Colour online) Droplet with viscous interface and $\lambda=1$. (a) Error in viscous force for a spherical droplet with $B q=B q_{s}=B q_{d}=1$ and infinite $C a$. The number of elements $N$ varies from 80 to 20480 . The order of convergence is one (solid line). (b) Variation with dimensionless time $\dot{\epsilon} t$ of the Taylor parameter $D$ with two sets of parameters $(C a ; \lambda ; B q)=(0.5 ; 1 ; 10)$ and $(C a ; \lambda ; B q)=(0.35 ; 0.1 ; 5)$ for three numbers $N$ of elements.

propose it as a benchmark problem. Indeed, Schwalbe's analysis establishes that the droplet's migration velocity for $\lambda=1$ is

$$
\frac{\boldsymbol{U}_{\text {analytic }}}{\alpha a^{2}}=-\frac{2 B q_{d}+3}{3\left(2 B q_{d}+5\right)} e_{y}
$$

for a droplet with radius $a$. Thus, as $B q_{d} \rightarrow \infty, \boldsymbol{U}_{m} / \alpha a^{2}$ tends toward $-(1 / 3) e_{y}$.

Thus, a spherical droplet is centred in a planar Poiseuille flow $v^{\infty}$,

$$
\boldsymbol{v}^{\infty}=\left(V-\alpha y^{2}\right) e_{x},
$$

for speed $V$ at the centre line and $\alpha$ being proportional to the flow profile's curvature (Schwalbe et al. 2011). The migration velocity $\boldsymbol{U}_{m}$ may then be defined as

$$
\boldsymbol{U}_{m}(t)=\frac{1}{\Omega(t)} \int_{\Omega(t)}\left(\boldsymbol{v}(\boldsymbol{x}, t)-\boldsymbol{v}^{\infty}(0)\right) \mathrm{d} \boldsymbol{x}
$$

for velocity $\boldsymbol{v}(\boldsymbol{x}, t)$ on the droplet, undisturbed velocity $\boldsymbol{v}^{\infty}(0)$ at the centre line and droplet volume $\Omega$ (Reusken \& Zhang 2013). We consider a capillary number $C a=0.01$ to maintain the deformations below $O\left(10^{-4}\right)$. The results were checked by another approach taking $C a=1$ but a very small time step of $10^{-5}$. The velocity is measured at short times preventing the appearance of any deformation (no inertia). There are no differences between the two calculations.

The instantaneous $\boldsymbol{U}_{m}$ was computed and the error is defined as $\left\|\boldsymbol{U}_{m}-\boldsymbol{U}_{\text {analytic }}\right\|_{2}$. A grid refinement study is shown in figure $9(a)$, for $B q_{d}=0,1$ and 10 . The convergence is of second order with respect to the number of triangular elements $N$. A comparison with analytic results for a broad range of $B q_{d}$ is given in figure $9(b)$, using a mesh with $N=320$ triangles. Again, excellent agreement occurs over the entire set of $B q_{d}$ considered. 

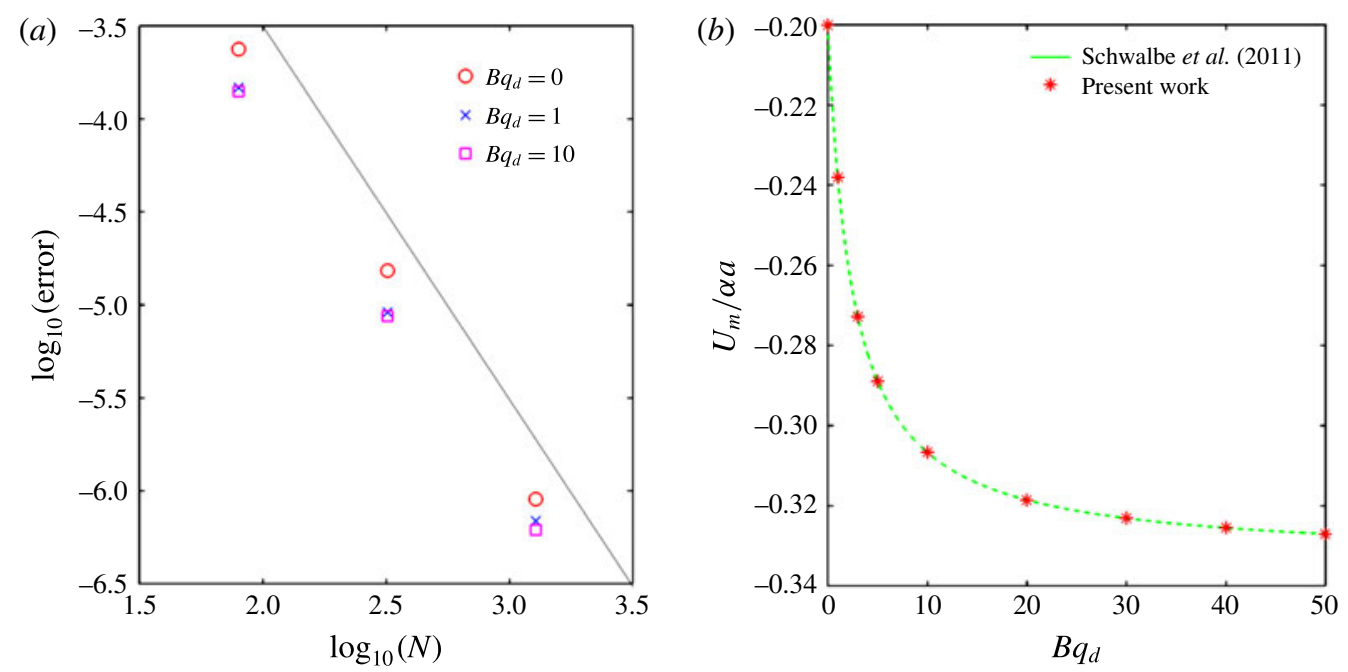

FIGURE 9. (Colour online) Droplet with viscous interface. (a) Error for $\boldsymbol{U}_{m} / \alpha a^{2}$ for $B q_{d}=0,1$ and 10 with a second-order convergence. (b) Computed $\boldsymbol{U}_{m} / \alpha a^{2}$ compared with Schwalbe et al. (2011).

\section{Results}

\subsection{Surface viscosity $B q=B q_{s}=B q_{d}$}

Large deformations of a droplet with identical shear and dilational surface viscosities were investigated numerically by Pozrikidis (1994), but steady-state results were difficult to compute. Accordingly, we compare in figure 10 the steady-state behaviour of droplets with surface viscosity $B q$ to the predictions from the small deformation analysis of Flumerfelt (1980) which is currently the only one taking into account the dilational and shear interface viscosities. Unless they are very heavily deformed, the steady-state shapes for droplets with surface viscosity are approximately ellipsoidal and, therefore, are well described by the Taylor deformation parameter $D$.

In the case of $\lambda=1$, results are shown for $C a=0.1,0.33$ and 0.5 . As with clean droplets, deformation increases with the capillary number. The first two capillary numbers belong to the stable region for clean droplets $C a<C a_{c}$, but the last is larger than $C a_{c}$ for a clean droplet. This clearly questions the contribution of interfacial viscosities to breakup: this will be discussed in more details below. The Taylor parameter $D$ decreases monotonically with the Boussinesq number whatever the capillary number: figure 10. Surface viscous dissipation is expected to reduce the deformation as also observed for high viscosity contrast. However, contrary to this basic argument, this result becomes false considering different weights for dilational and shear Boussinesq numbers $\left(B q_{s} \neq B q_{d}\right)$ as we will show below. We return to the case $B q_{s}=B q_{d}$. With $C a=0.1$, the smallest deformations occur and the agreement between the present method and Flumerfelt (1980) for $D$ is excellent except at zero $B q$. Even at the two higher capillary numbers, agreement is surprisingly consistent. In the limit of large Boussinesq number, $B q \geqslant 30$, the inclination and the Taylor parameter are in excellent agreement with Flumerfelt (1980) whatever the capillary number. Only as $B q \rightarrow 0$ does divergence emerge for $D$, reflecting the known disparity for clean droplets. It is striking that small deformation theory describes droplets with viscous surfaces much better than clean droplets. 

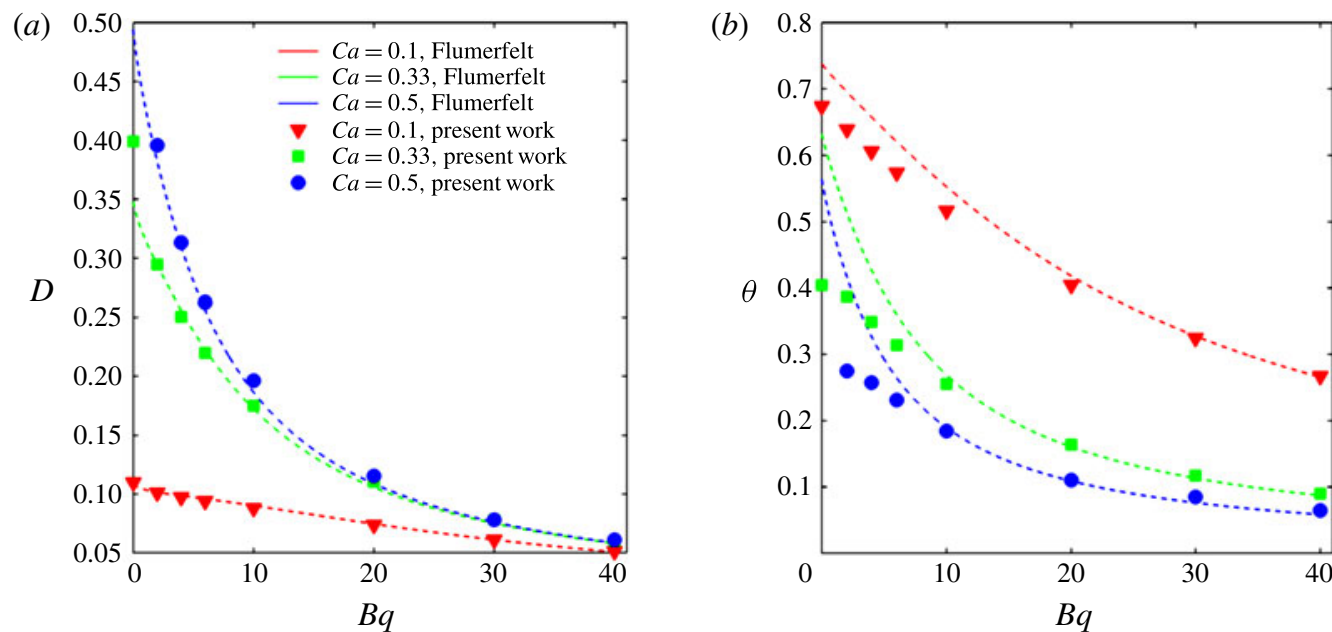

FIgure 10. (Colour online) Steady-state $(a)$ deformation and $(b)$ inclination angle of droplets with surface viscosity $B q$ in shear flow, compared with Flumerfelt (1980) in the case $\lambda=1$.

However, a more obvious disagreement with small deformation theory is observed for inclination angle $\theta$. At moderate $B q \leqslant 10, \theta$ is not well described contrary to $D$ (figure 10), recovering the discrepancy of the Cox model to model the deformation of clean droplet in shear flow (see figure 6 and the validation part). Unfortunately, there is no theory for droplets with surface viscosities equivalent to the model of Chaffey \& Brenner (1967) for the first-order variation of the inclination with $\mathrm{Ca}$ with the slope depending on $\lambda$ and $B q$, or to the more accurate models with expansion to the third order with $\mathrm{Ca}$ (Vlahovska et al. 2005, 2009a). For larger Boussinesq number, $B q \geqslant 15$, the present results and small deformation theory begin to agree nicely for the inclination angle. As with deformation, this agreement at large $B q$ is better than previous studies of clean droplets would suggest, which is at least partly due to the smaller deformations incurred at larger $B q$. It is observed that a droplet with surface viscosity has a lower steady-state angle of inclination than the corresponding clean droplet. Generally, $\theta$ decreases monotonically as $B q$ is increased. As observed for clean droplets with large $\lambda$ (Kennedy et al. 1994), we see that the droplet's major axis tends toward the axis of flow due to viscous dissipation. Consequently, inclination angles are smallest for higher $\mathrm{Ca}$, as viscous dissipation on the surface dominates the surface stress of these droplets.

The impact of surface viscosity is also clearly represented in the flow pattern on the droplet. Surface viscosity tends to decrease any interfacial gradient of velocity. In the plane of flux, $x y$-plane, the surface (and inner) flow becomes almost circular while in the $x z$-plane, the streamlines are parallel and the magnitude of velocity varies moderately with $z$ on a larger depth compared to a clean droplet. The steady-state velocity magnitudes of droplets with clean and viscous surfaces at $C a=0.33$ are depicted in figure 11 . In the case $\lambda \neq 1$, the deformation of droplets with a viscous interface was studied on six decades of viscosity contrast from $10^{-3}$ to $10^{3}$, five Boussinesq numbers $B q$ from 0 to 50 and four capillary numbers $C a$ from 0.1 to 0.5 : figure 12. We recover the same typical variation of the Taylor parameter as for clean droplets characterized by a plateau at small viscosity contrast and an asymptotic weak 

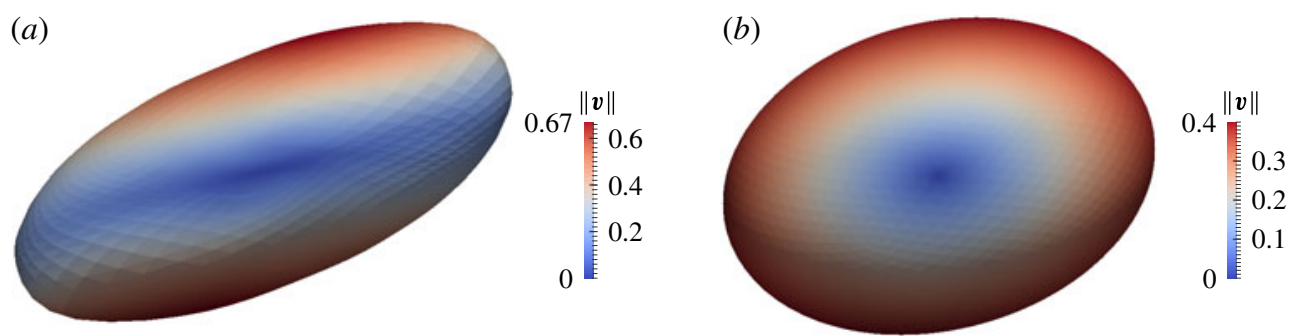

FIGURE 11. (Colour online) Magnitude of velocity $\|\boldsymbol{v}\|$ on droplet surface in steady state with $\lambda=1$. (a) Droplet with $C a=0.33$ and $B q=0$. (b) Droplet with $C a=0.33$ and $B q=10$.
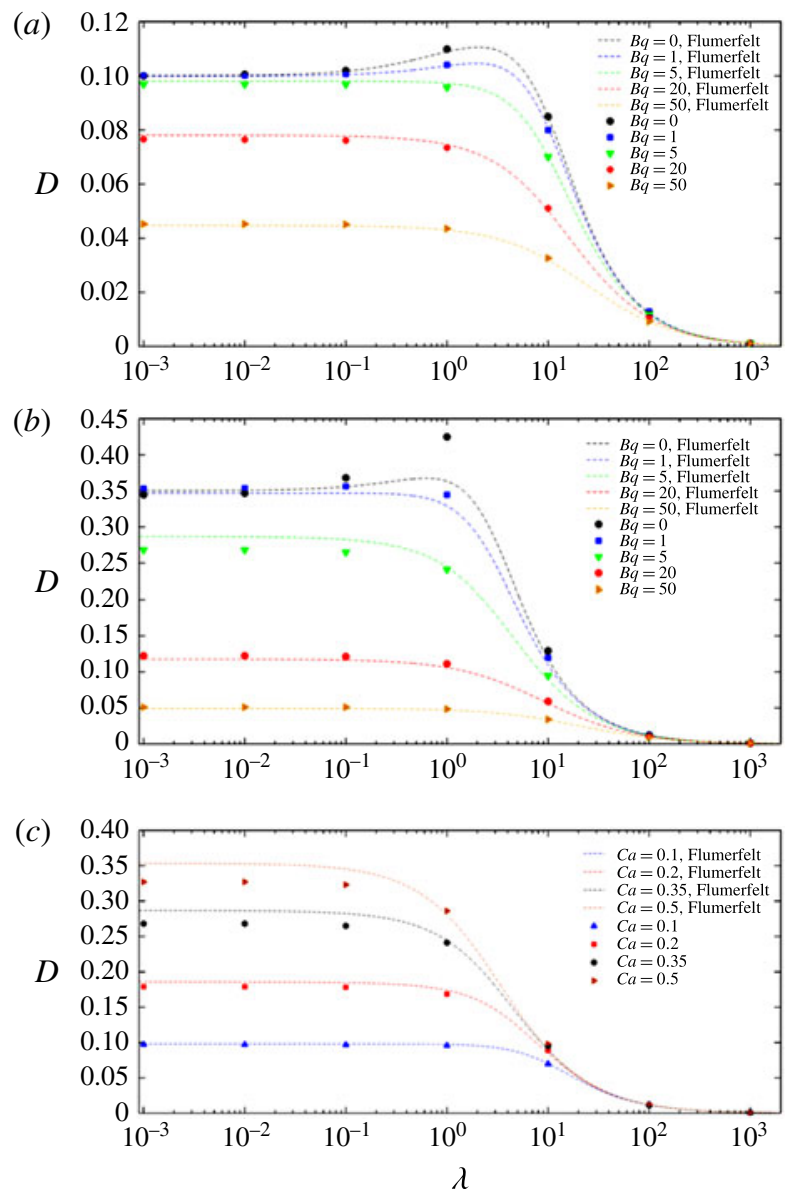

FIGURE 12. (Colour online) $(a, b)$ Deformation of a droplet with viscous interface in a shear flow as a function of the $B q$ and $\lambda$. (c) Deformation of a droplet as a function of $C a$ and $\lambda$. (a) $C a=0.1$. (b) $C a=0.35$. (c) $B q=5$. 
decreasing for viscous droplets. In the limit of $B q \rightarrow 0, D$ reaches a maximum in the moderate range of viscosity contrast, namely $\lambda=0(1)$. For $B q \geqslant 1$, it disappears. At small capillary number $C a=0.1$ characterized by small deformations, there is a good agreement with the theory of Flumerfelt (1980) on all the range of viscosity contrast and Boussinesq number. The agreement is also excellent for viscosity contrast larger than 100 whatever $C a$ and $B q$. For $C a=0.35$, the agreement is very good for $B q \geqslant 20$ : figure $12(b)$. For higher capillary numbers, the approximation of small deformations is not satisfied and the deviation from theory appears clearly: figure 12(c). As expected, the theory of Flumerfelt (1980) is useful in the case of small deformations which can be reached under a moderate capillary number or a high surface viscosity.

Thus far, we have studied the stationary state of a stable droplet with a viscous interface and viscosity contrast in shear flow: a tank-treading motion of the interface with a stationary shape. Here, the dynamics to reach this state is explored. This is particularly important to interpret correctly experimental results.

Kennedy et al. (1994) note that, for $\lambda>\lambda_{c}$, a damped oscillation in $D$ and $\theta$ occurs during the droplet's approach to steady state. In contrast, a droplet with a smaller $\lambda$ undergoes a smooth, if not necessarily monotonic, transition to equilibrium. Oscillations occur when the flow time scale and droplet relaxation time scale differ significantly. The shorter time scale causes oscillations by 'over-shooting', while the longer time scale gradually damps this phenomenon and steady state is eventually achieved. Experiments in Erni, Fischer \& Windhab (2005) also indicate similar oscillations for droplets with a viscoelastic surface layer.

At higher values of $\beta=C a B q$ in our simulations, a similar transient oscillating relaxation is observed: figure $13(a, b)$. The change between transient oscillating and consistent tank-treading occurs near $\beta=3.5$ as highlighted by figure $13(d)$. At a given $C a$, increasing $B q$ results in an increased oscillation frequency. For large $B q$, the oscillation frequency becomes nearly independent of $C a$, as shown in figure $13(a, b)$ for $B q=40$. In fact, two modes of relaxation to tank-treading can be distinguished. For $3.5 \leqslant \beta \leqslant 10$, the longest axis of the droplet oscillates around a positive inclination. In this range, the criterion is that the temporal variations of Taylor parameter and inclination $\theta$ have at least both an overshoot followed by an undershoot. Above $\beta \geqslant 10$, the inclination oscillates between negative and positive values. The curve $\beta=10$ has been determined by the additional criterion $\theta \leqslant 0$ for some time. In the limiting case of $C a=\infty$, as predicted by Barthès-Biesel \& Sgaier (1985), the droplet displays an undamped oscillation between $\theta=\pi / 4$ and $-(\pi / 4)$.

Despite their similar frequencies, the amplitude and decay rate of the oscillations shown differ quite significantly, with the persistence of the oscillation increasing with $\mathrm{Ca}$. However, if time $t$ is normalized by surface tension time scale $\tau_{\gamma}=\left(\mu^{e x t} a / \gamma\right)$, figure 13(c) shows that the amplitudes of the oscillations have the same exponential rate of decay. Thus, transient oscillating behaviour occurs when surface viscosity dominates surface tension, but the properties of this oscillation depend on each quantity separately. As with $\lambda$ in Kennedy et al. (1994), tumbling-like behaviour is not observed for spherical droplets for any value of $B q$. However, an initially prolate spheroidal droplet will tumble transiently if the major axis is initially inclined relative to the direction of flow.

Overall, presence of additional dissipation due to surface viscosity $B q$ leads to a smaller deformation than would be observed for the corresponding clean droplet, as shown for droplets with large $\lambda$ (Kennedy et al. 1994). As Bq becomes large, the three semi-axes tend to the same value, the droplet becoming spherical, almost independently of $\mathrm{Ca}$. Further, the presence of surface viscosity $B q$ raises the $C a_{c}$ 

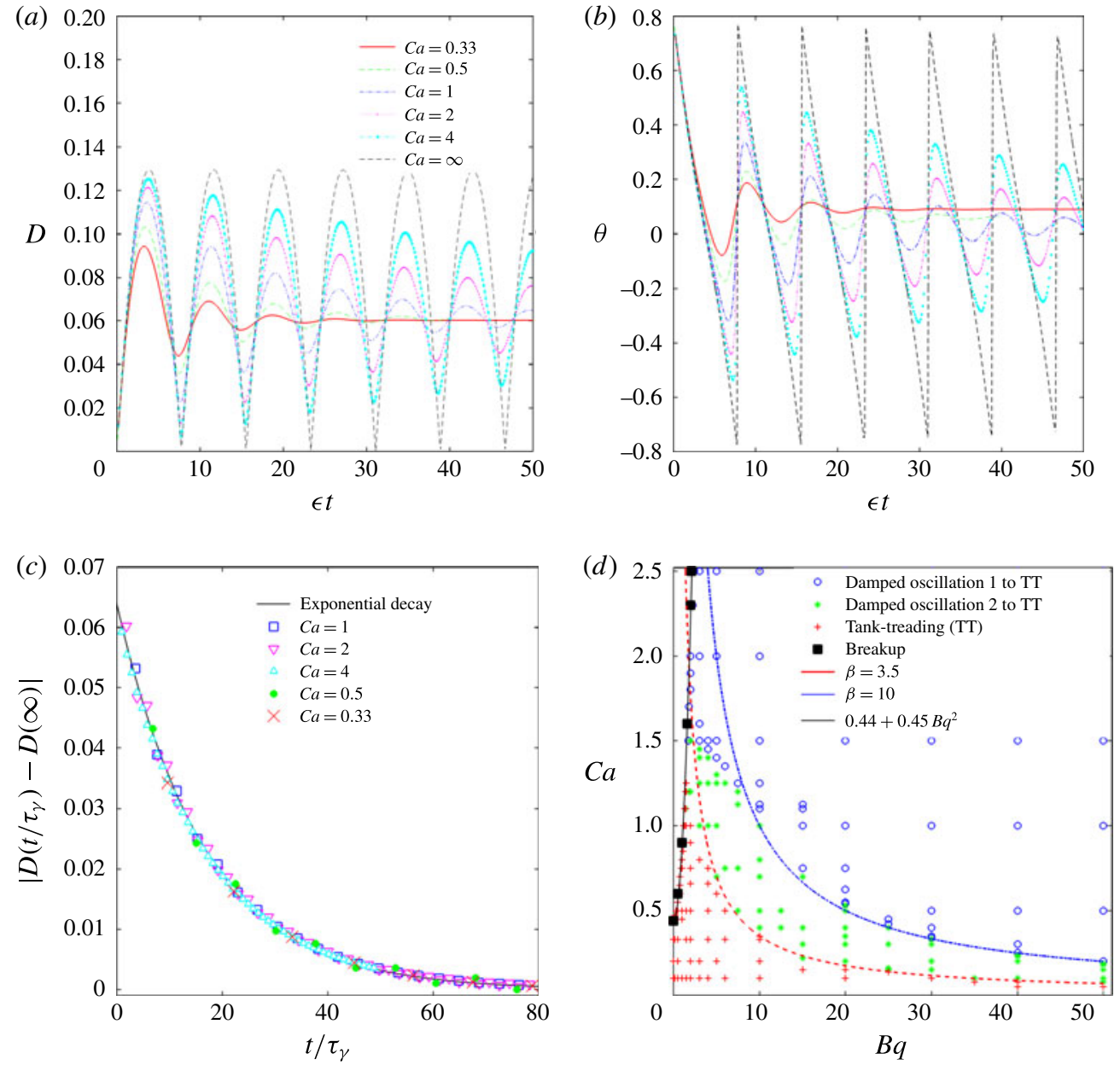

FIGURE 13. (Colour online) Droplet with surface viscosity and $\lambda=1$. $(a, b)$ The droplet shape relaxes to its tank-treading shape by numerous oscillations $(B q=40)$. Larger is the capillary number, longer is the persistence time of oscillations. (c) The amplitude of transient oscillatory relaxation to tank-treading motion decreases exponentially with a viscous characteristic time $\tau_{\gamma}=\mu^{e x t} a / \gamma$ based on surface tension $(B q=40)$. (d) A droplet with viscous interface has a tank-treading (TT) motion or evolves to breakup. The two domains are separated by the continuous black line in the space of parameters $(B q ; C a)$. In the domain of TT, the longer axis can oscillate transiently around a positive inclination angle (damped oscillation 2, green disc) or begins to oscillate between $-\pi / 4$ and $\pi / 4$ to return more slowly to an oscillation around a positive inclination (damped oscillation 1, blue circles). For $B q<2$, the different relaxations are not distinguished for the sake of clarity.

above the value for a clean droplet, as steady states were computed at $C a=0.5$ in figure 10. The dependence of $C a_{c}$ is examined in more details below.

To determine the influence of $B q$ on the stability of a droplet under shear flow, we compute the dynamics of a initially spherical droplet immersed in a equiviscous $(\lambda=1)$ shear flow abruptly started. The droplets are then classified as stable if their shape tends towards an (elongated) ellipsoid (cf. figure 14a) and unstable if 
(a)

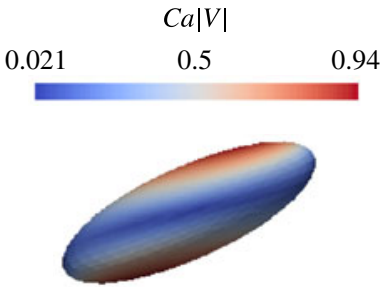

(b)

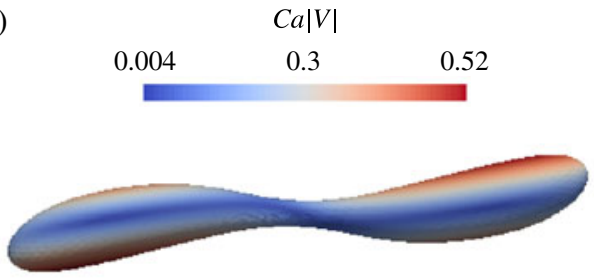

FIgURE 14. (Colour online) Droplet with surface viscosity $B q=0.1$ and $\lambda=1$. (a) The shape is stationary. (b) The shape is unstable and the drop evolves to breakup. (a) $\mathrm{Ca}=$ 0.45. (b) $C a=0.47$.

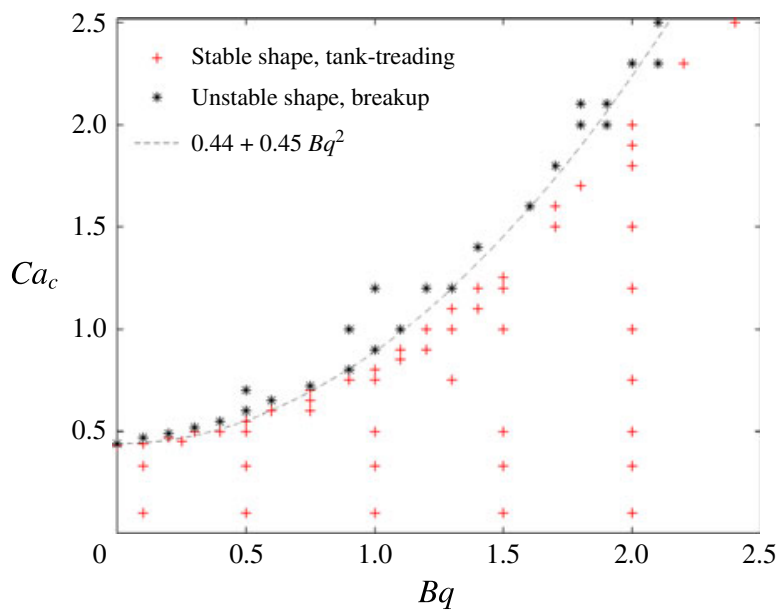

FIGURE 15. (Colour online) Breakup diagram in the case $\lambda=1$. Droplet shapes are unstable above a critical capillary number $C a_{c}$ which increases with the Boussinesq number $B q$. The parabolic dashed line $-C a_{c}=0.44+0.45 B q^{2}-$ is a guide for eye providing a good fit of the transition from tank-treading stable shapes to breakup.

their shape tends towards an unsteady characteristic shape composed of two bulbous ends connected by a neck (cf. figure 14b). The figure 15 shows the nonlinear variation of the critical capillary number with the Boussinesq number. Note that this is reminiscent of previous studies (Kennedy et al. 1994; Li et al. 2000; Cristini et al. 2003) which have investigated the dependence of $C a_{c}$ on $\lambda$, observing an intriguingly nonlinear relationship. Further, these studies define a critical fluid viscosity ratio $\lambda_{c}$, beyond which all clean droplets have stable equilibria, independently of $\mathrm{Ca}$. Here, a critical Boussinesq number beyond which no breakup can occur also exists, since the oscillating dynamics is permanent for $C a=\infty, B q=40$.

\subsection{Respective roles of shear and dilational surface viscosities}

Flumerfelt (1980) considers several scenarios concerning the effects of surface viscosity, including when $B q_{s}$ or $B q_{d}$ is large relative to $C a, \lambda$ and the other Boussinesq number. In contrast to the trend for large $B q$, the deformation and angle of inclination approach non-zero limits as either $B q_{s}$ or $B q_{d} \rightarrow \infty$. Further, Flumerfelt notes that, for small capillary numbers, $B q_{s}$ and $B q_{d}$ becoming large leads 


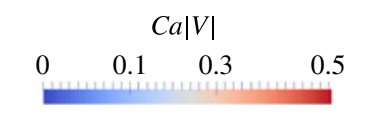

(a)

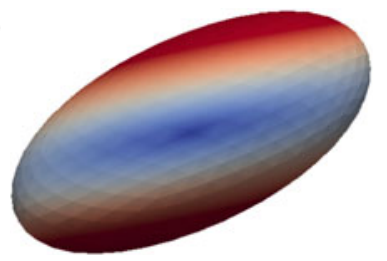

(c)

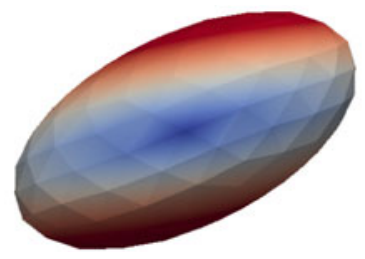

(e)

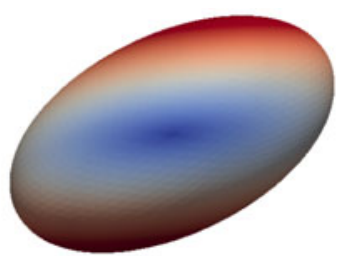

(g)

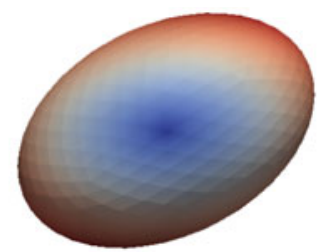

$\mathrm{Ca}|\mathrm{V}|$

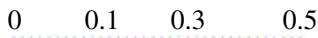

(b)

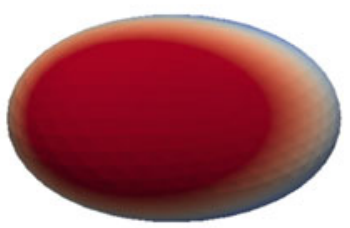

(d)

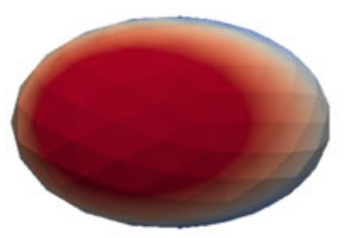

$(f)$

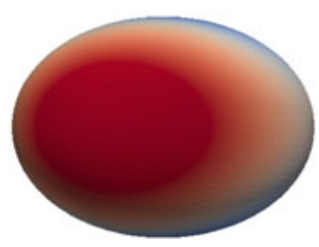

(h)

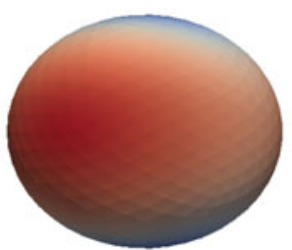

FIGURE 16. (Colour online) A droplet with various shear viscous surface $B q_{s}, \lambda=1, C a=$ 0.3 and $B q_{d}=0$ in shear flow. $(a, c, e, g)$ Corresponds to the flow plane $(x, y)$ while $(b, d, f, h)$ to the view $(x, z)$. $(a, b) B q_{s}=0,(c, d) B q_{s}=0.3$, (e,f) $B q_{s}=1,(g, h) B q_{s}=5$.

to smaller and larger deformations, respectively. However, as Flumerfelt's analysis is limited to small deformations and steady-state values, it is interesting to consider how $B q_{s}$ and $B q_{d}$ separately alter a droplet's dynamics in shear flow.

\subsubsection{Shear surface viscosity $B q_{d}=0$}

An example of the steady-state deformation and inclination angle for droplets is shown in figure 16 where only the value of shear surface viscosity is varied from 0 to 10. The shapes appear more quasi-spherical ( $D$ decreases) as the shear Boussinesq number increases. As for the case $B q=B q_{s}=B q_{d}$, this result is expected due to a stronger droplet dissipation. This result is confirmed by a study of shape deformations for three capillary numbers $C a=0.1,0.33,0.5$ with $0 \leqslant B q_{s} \leqslant 40$. At small deformation $(\mathrm{Ca}=0.1)$, the Taylor parameter is in good agreement with Flumerfelt (1980) while it is not the case for higher capillary numbers even if 

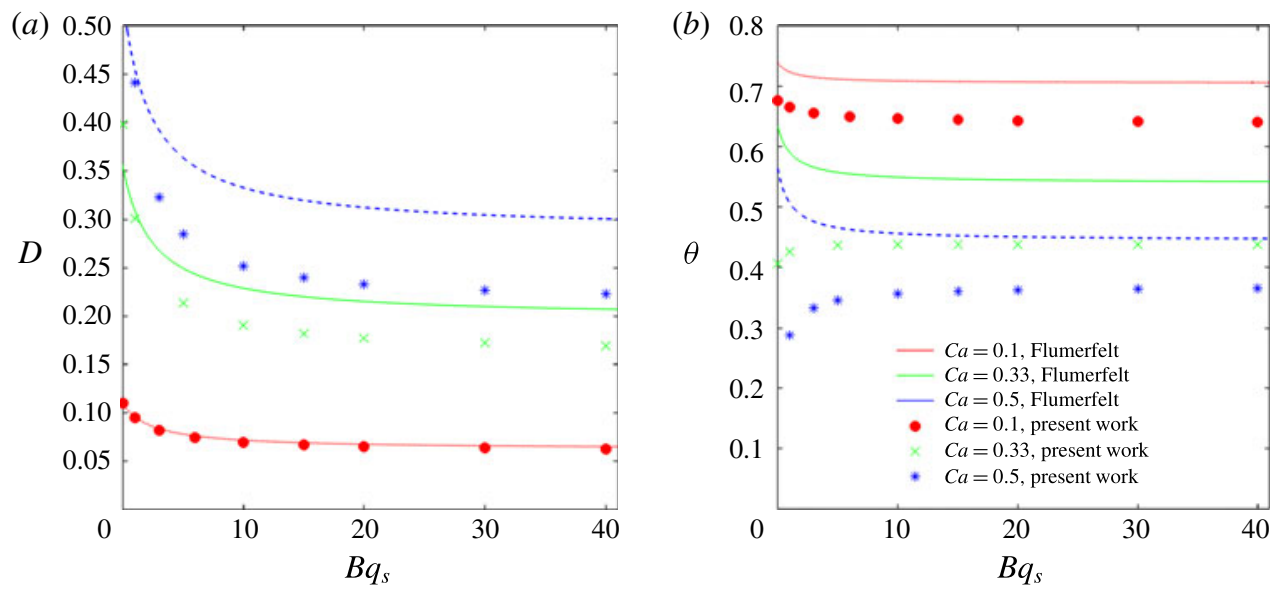

FIGURE 17. (Colour online) Droplet with surface viscosity and $\lambda=1$. Steady-state deformation $(a)$ and inclination angle $(b)$ of droplets with shear surface viscosity $B q_{s}$ in shear flow, compared with Flumerfelt (1980).

(a)

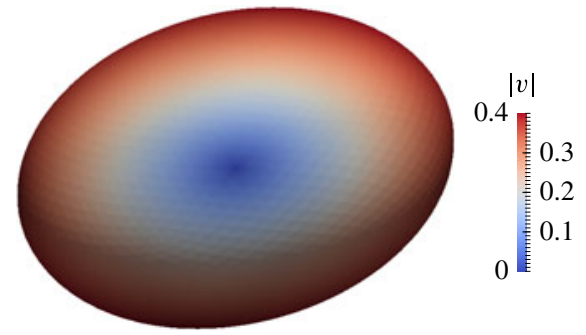

(b)

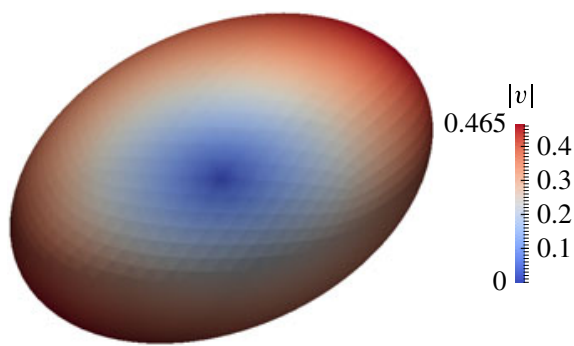

Figure 18. (Colour online) Comparison between a droplet with $C a=0.33$ and $\lambda=1$ in shear flow. (a) $B q_{s}=B q_{d}=10$, (b) $\left(B q_{d} ; B q_{s}\right)=(0 ; 10)$.

the trend is recovered: figure $17(a)$. On the contrary, the effect of shear surface viscosity has a weaker impact on the inclination angle which is not well described by Flumerfelt (1980) quantitatively and qualitatively. Indeed, in the limit of small to moderate $B q_{s}$, the inclination increases with $B q_{s}$ for high capillary numbers, contrary to theory: figure $17(b)$. At small capillary number $(C a=0.1)$, the classic decreasing of the inclination with the shear viscosity is recovered. But, the values reached at large shear Boussinesq number are overestimated by theory for all capillary numbers.

More importantly, the stable steady states at $C a=0.5$ in figure 17 show that purely shear surface viscosity is sufficient to increase the critical capillary number of a droplet. This result is consistent with previous suggestions of a correlation between shear surface viscosity and stability. Note that in the next section, we show that the dilational viscosity is not able to stabilize the shape above the critical capillary number for a clean droplet, contrary to the shear viscosity. Further, a comparison of shapes and magnitude of velocity in figure 18 indicates that $B q$ and $B q_{s}$ Boussinesq numbers lead to similar velocity fields on the droplet but to different inclinations.

Moreover, droplets with purely shear surface viscosity $B q_{s}$ lack a characteristic behaviour noted for droplets with $B q$. The magnitude of $B q_{s}$ does not alter the time required for the droplet to reach steady state; indeed, as shown and discussed further 

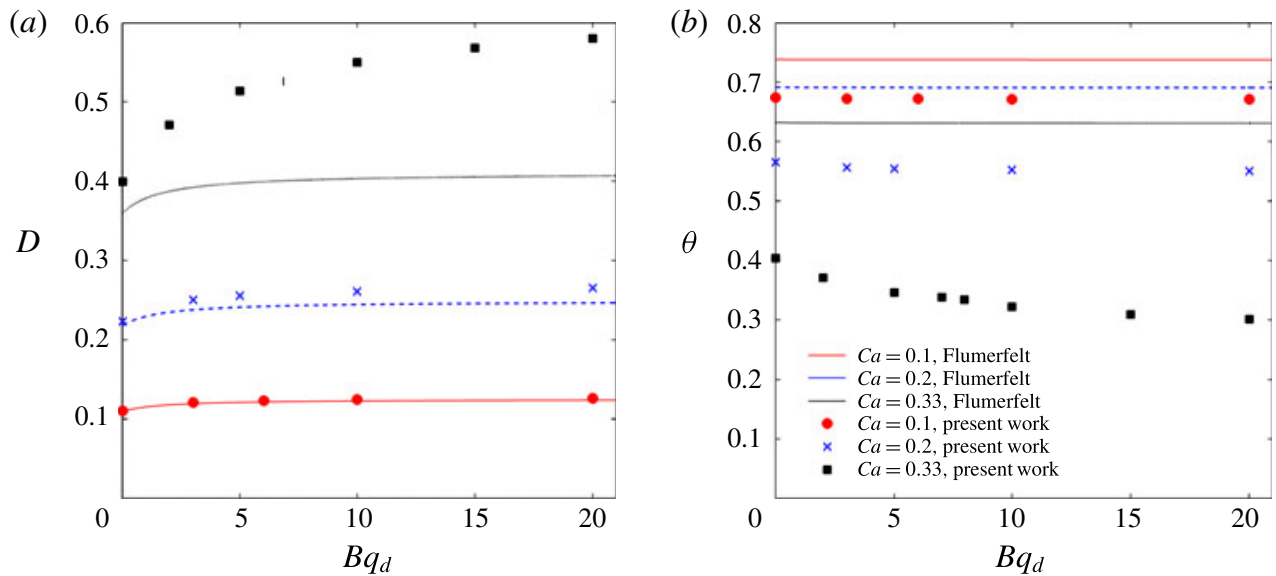

FIGURE 19. (Colour online) Droplet with surface viscosity and $\lambda=1$. Steady-state deformation $(a)$ and inclination angle $(b)$ of droplets with dilational surface viscosity $B q_{d}$ in shear flow, compared with Flumerfelt (1980).

(figure 21), the time is comparable to the time for clean drops with the same $\mathrm{Ca}$. On the contrary, transient oscillatory relaxation to tank-treading motion is still observed but at high values of $B q_{s}$. In a shear flow characterized by $C a=0.1$, oscillations are visible for $B q_{s} \geqslant 10^{4}$, which is out of the experimental range for droplets.

\subsubsection{Dilational surface viscosity $B q_{s}=0$}

The expected role of purely dilational surface viscosity is somewhat clearer, as Flumerfelt incorporated both dilational surface viscosity and small variations in surface tension into a single apparent surface dilational viscosity. Computations and experiments with surfactant concentrations on the droplet surface have shown the major axis is elongated, while both minor axes become smaller (Feigl et al. 2007). As a result, the droplet deformation $D$ may significantly increase and, therefore, decrease a given drop's critical capillary number $C a_{c}$.

The steady-state deformation and inclination angle for drops with only dilational surface viscosity $B q_{d}$, are compared with the small deformation analysis from Flumerfelt (1980) in figure 19. As in previous cases, the present method agrees well with Flumerfelt for $C a=0.1$ (and, to a lesser extent, 0.2), excepting the standard overprediction of the inclination angle. At $C a=0.33$, however, our results show a much more significant increase in $D$ and, indeed, a steady-state equilibrium does not exist beyond $B q_{d}=200 \pm 40$. Thus, surface dilational viscosity is effective in decreasing the critical capillary number of a drop. Consistently, steady-state values for dilational surface viscosity at $C a=0.5$ or other high capillary numbers do not exist: all droplets in this case tend to breakup.

Studies of surfactant transport have noted two processes, convection and dilution, which serve to increase and decrease droplet deformation, respectively (Feigl et al. 2007). For dilational surface viscosity, the analogous terms are negative and positive surface velocity divergence, respectively. Distributions of the surface velocity divergence for droplets with dilational surface viscosity are shown in figure 20. In particular, the negative velocity divergence at the ends of the major axis serves to lower the surface stress in those regions and leads to larger droplet deformation. 
(a)

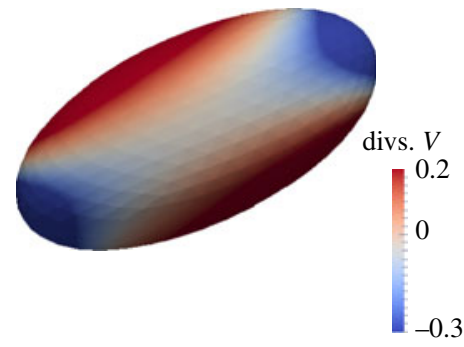

(c)

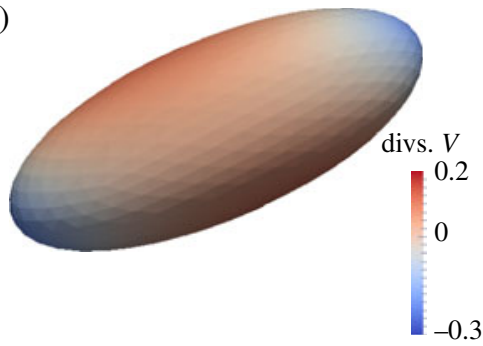

(b)

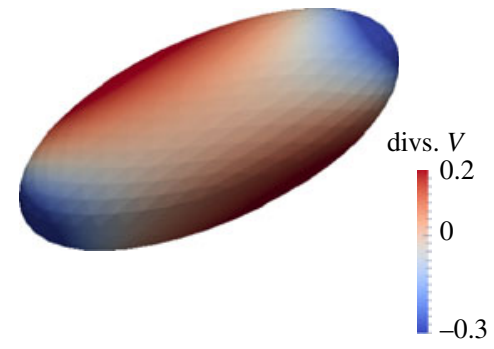

(d)

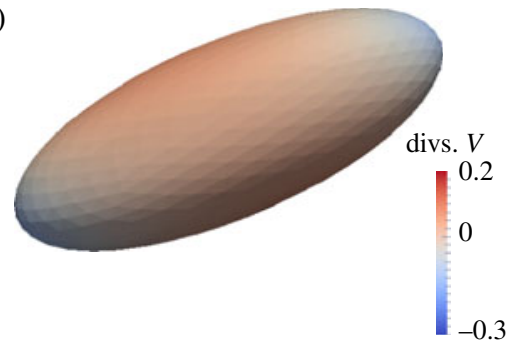

FIGURE 20. (Colour online) Effect of dilational surface viscosity on the droplet shape (longest axis increasing) and variation of surface divergence of membrane velocity in steady state. Here, $B q_{s}=0$ and $\lambda=1$. (a) $\nabla_{s} \cdot \boldsymbol{v}$ with $C a=0.3$ and $B q_{d}=0$, (b) $\nabla_{s} \cdot v$ with $C a=0.3$ and $B q_{d}=1,(c) \nabla_{s} \cdot v$ with $C a=0.3$ and $B q_{d}=5,(d) \nabla_{s} \cdot v$ with $C a=0.3$ and $B q_{d}=10$.

(a)

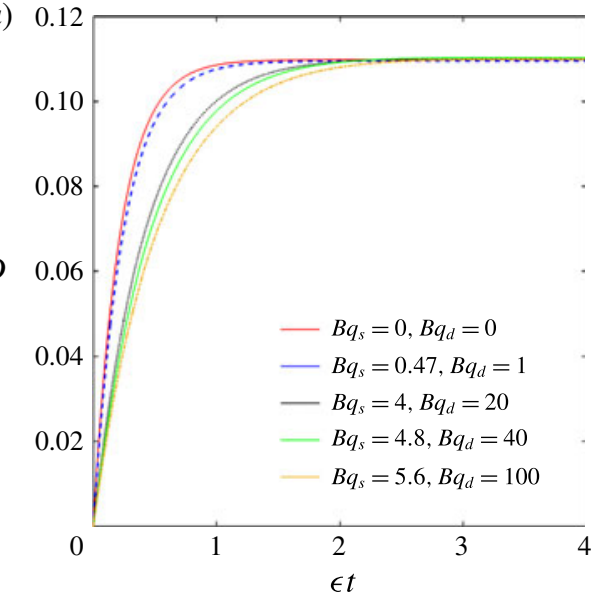

(b)

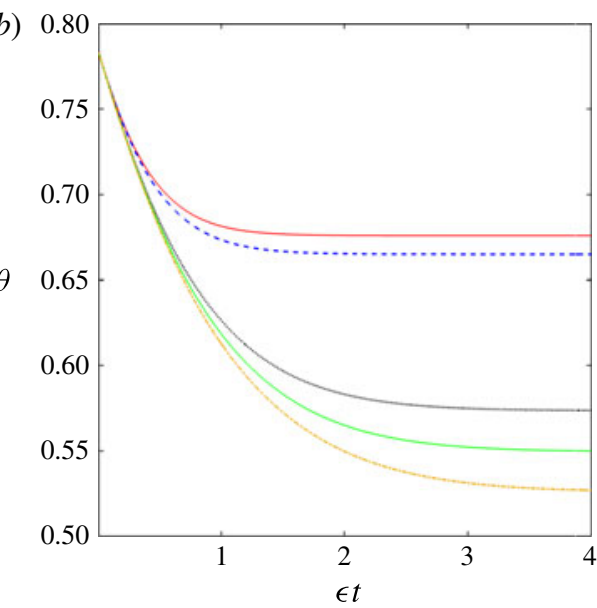

Figure 21. (Colour online) Droplet in a shear flow $C a=0.1$ with $\lambda=1$ for five combinations of shear $B q_{s}$ and dilational $B q_{d}$ surface viscosities but with the same deformation, namely the Taylor parameter $(a)$. On the contrary, the inclination angles are different $(b)$.

At $C a=0.1$, this effect is limited by the high $\gamma$ and by the positive velocity divergence about the equator of the droplet. At $C a=0.3$, however, both of these limiting factors are reduced and negative surface velocity divergence dominates, leading to significantly larger deformation even at $B q_{d}=1$ : figure 20. Dilational 


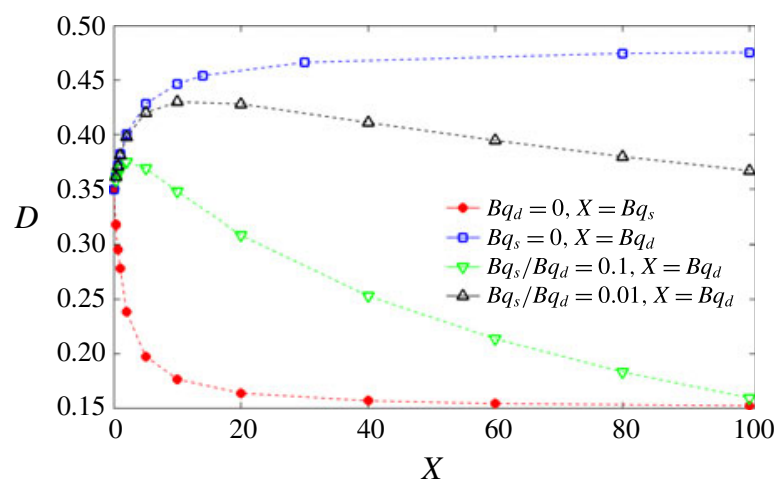

FIgURE 22. (Colour online) Steady-state deformations of a droplet with $\lambda=1$ in shear flow $(C a=0.1)$ with various shear and dilational surface viscosities.

surface viscosity does not have a large qualitative effect on the velocity distribution on the droplet but does decrease the velocity magnitude, as also observed for Marangoni stress (Feigl et al. 2007).

As with shear surface viscosity alone, aspects from simulations with both surface viscosities were lacking here. No transient oscillating relaxation to permanent tanktreading shape was observed as drops approached steady state, but dilational surface viscosity did slightly increase the time required to reach steady state.

\subsubsection{Shear and dilational surface viscosities}

An interesting consequence of shear and dilational surface viscosity tending to decrease and increase deformation, respectively, is that certain combinations of surface viscosity will lead to the same steady-state deformation as the corresponding clean droplet (albeit at a slightly different angle of inclination). For instance, in figure 21(a), four combinations of $B q_{s}$ and $B q_{d}$ result in the same deformation as a clean droplet at the same capillary number: $D \approx 0.11$. In figure 21 , it is striking that the dilational surface viscosity varies by a factor 100 while the shear surface viscosity by a factor 10 approximately. This prevents a measurement of Boussinesq numbers by only the Taylor parameter. However, following the theoretical work of Flumerfelt (1980), Phillips et al. (1980) proposed that experimentally-determined deformation and inclination simultaneously may be used to solve for a droplet's surface viscosity coefficients. We checked that the inclination angle varies for these five couples on a reasonable range: figure $21(b)$. Our results for purely shear or dilational surface viscosity suggest a limitation of this approach. Indeed, at $C a=0.1$, for the same Taylor parameter, the inclination varies smoothly with $B q_{s}$. Such a measurement by inverse method needs a careful interplay between experiments and numerical simulations, limiting its widespread use. Moreover, when $B q_{s} \gg 1$ and $B q_{d} \ll 1$, deformation and inclination angle are unlikely to differ by a sufficient amount to make an accurate estimation. To confirm the complex dependence of deformations in respect of the shear surface viscosity for a droplet in shear flow, we performed also the study of deformations by setting the ratio $B q_{s} / B q_{d}$ to $0,1 \%, 10 \%$ and $\infty\left(B q_{s}=0\right)$ : see the figure 22. First, we recover the results of previous parts with only one Boussinesq number: $B q_{s}$ (red curve) decreases the deformation while $B q_{d}$ increases it (blue curve). In the cases of $1 \%$ or $10 \%$, the deformations begin to increase and reach a maximum for approximately $B q_{d}=2$ and $B q_{d}=10$, respectively. 
Despite this behaviour at small to moderate $B q_{d}$, the deformations of pure shear and $B q_{s} / B q_{d}=10 \%$ become equal for $B q_{d}=100$. It can be inferred that the deformation is not a function of a linear combination of $B q_{s}$ and $B q_{d}$. We recover this phenomenon for a minute contribution of shear surface viscosity, namely $1 \%$ : figure 22 . It means notably a strong dependence on $B q_{s}$ when $B q_{d} \gg 1$.

\section{Conclusion}

This paper presents a numerical study of the effect of surface viscosities on droplet dynamics in shear flow performed with a new numerical method. The droplet surface is described with a Loop subdivision method, which provides the necessary continuity to compute the surface rate of deformation tensor and Boussinesq-Scriven stress tensor. The interface motion is calculated by the boundary integral method associated with a RK45 temporal scheme. While derived for droplet surfaces, the method is naturally extensible to capsule and vesicle models. When combined with the boundary element model, to describe the bulk Stokes flow and fluid-interface interaction, the method is used to simulate the response of an immiscible droplet with a viscous surface to deformation in shear flow.

Validation and convergence analysis of the method is presented by comparing with previous analytical and numerical works in the case of a clean (no surface viscosity) droplet with viscosity contrast. The method is then used to compute the influence of equal shear and dilational surface viscosities, measured by the Boussinesq number $B q=B q_{s}=B q_{d}$. Surface viscosity is shown to decrease deformation and the angle of inclination. Numerical results are systematically compared to the small deformation analysis of Flumerfelt (1980), allowing us to discuss its range of validity: notably, the deformation parameter is very well captured by theory, except in the limit $B q \rightarrow 0$, while the inclination angle is only qualitatively described, the theory matching the numerical results in the high surface viscosities limit $B q>30$. Surface viscosities not only affect the steady-state results, but also alter the dynamics of a drop under abruptly started shear flow. In particular, a transient damped oscillation is observed, persisting indefinitely in the limit of infinite capillary number. Finally, one crucial effect of surface viscosities is to strongly modify the critical capillary number: a drop with a slight surface viscosity $(B q=O(1))$ is much more stable than a clean drop. In other soft matter particles with surface viscosity, such as protein covered droplets, vesicles and red blood cells, shear Boussinesq numbers $B q_{s}=O(1)-O(10)$ are quite reasonable (Chang \& Olbricht 1993; Erni 2011). The recent work of Zell et al. (2014) on shear surface viscosity of surfactants shows that $\mu_{s}<10^{-2} \mu \mathrm{N} \cdot \mathrm{s} \mathrm{m}{ }^{-1}$, in contrast to previous studies that proposed values several orders of magnitude larger (Dickinson et al. 1988; Harvey et al. 2005). Even for relatively small droplets and a low bulk viscosity, this suggests that $B q_{s}<1$. Thus, while we find that shear surface viscosity in itself increases droplet stability, the effect at $B q_{s}<1$ is minor at best. On the other hand, even prior to Zell et al. (2014), it has been argued that $\mu_{s} \ll \mu_{d}$ (Georgieva et al. 2009), suggesting $B q_{s}$ and $B q_{d}$ values closer to the scenario considered in $\S 4.2 .2$. However, this statement is not correct. The results of the part $\S 4.2 .3$ have shown that even if $\mu_{s} / \mu_{d} \approx 0.01$, the effect of shear surface viscosity can dominate, at least for a droplet in shear flow. Further inquiry, taking into account surfactant transport, would be useful in clarifying both the relative magnitudes of the surface viscosities and their relation to emulsion stability. Numerical simulations of the full problem and comparisons with experiments will allow to determine these essential parameters to understand and control complex interface stability. 
A first step towards such understanding is to delineate the role of shear and dilational viscosities. Simulations presented in this paper show that the shear viscosity stabilizes droplet shapes and leads to overall lower deformation, while the dilational viscosity has the opposite effect, leading to increased elongation and thus reduced stability. Moreover, we found that these opposing effects could be combined in order to lead to a similar steady-state deformation for very different pairs of values of $\left(B q_{s}, B q_{d}\right)$. This highlights the strikingly complex role of rheological interfacial properties on the droplet dynamics.

If studies on the contribution of interfacial viscosities to droplet dynamics are scarce, the effect has been considered in the context of viscoelastic capsules by Yazdani \& Bagchi (2013). If a careful comparison is well beyond the scope of this paper, some general comments may be made in light of our own preliminary results. The damped oscillations observed here for droplets at large $\beta$ also occur for viscoelastic capsules with a high ratio of surface viscosities to shear elasticity, in both our preliminary results and in Yazdani \& Bagchi (2013). We also observe convergence to the results of Barthès-Biesel \& Sgaier (1985), in the limit of high $B q$ and vanishing shear elasticity. A subsequent paper will explore viscoelastic capsules in detail.

\section{Acknowledgements}

This work has benefited from financial support from the ANR Polytransflow (grant no.13-BS09-0015-01), from Labex MEC (grant no. ANR-11-LABX-0092), from A*MIDEX (grant no. ANR-11-IDEX-0001-02) and from CNES. This work was granted access to the HPC resources of Aix-Marseille Universite financed by the project Equip@Meso (ANR-10-EQPX-29-01).

\section{Appendix A}

The small deformation analysis of Flumerfelt (1980) studied the deformation $D$ and inclination $\theta$ of a spherical droplet in linear Stokes flows. Based on spherical harmonics, the expansion solution is first order in terms of the perturbation parameter. The method is based on the work of Cox (1969). The analysis considers a droplet with a viscosity contrast $\lambda$, along with constant non-dimensional shear and dilational surface viscosities $B q_{s}$ and $B q_{d}$, and small variations in surface surfactant concentration.

The modified viscosity ratio $\lambda^{\prime}$ is defined as

$$
\lambda^{\prime}=\lambda+\frac{6}{5} B q_{d}+\frac{4}{5} B q_{s} .
$$

Then, in shear flow, the steady-state deformation and inclination of a droplet with surface viscosity are given by Flumerfelt's equations (145) and (150):

$$
\begin{gathered}
D=\frac{1}{16} \frac{19 \lambda^{\prime}+16+\frac{6}{5} B q_{d}-\frac{36}{5} B q_{s}}{\left(\lambda^{\prime}+1\right)\left(C a^{-2}+\left(\frac{19}{20} \lambda^{\prime} R\right)^{2}\right)^{1 / 2}} \\
\theta=\frac{\pi}{4}-\frac{1}{2} \arctan \left(\frac{19 C a \lambda^{\prime} R}{20}\right)
\end{gathered}
$$

in which

$$
R=1-\frac{1}{114 \lambda^{\prime}}\left(\frac{678}{5} B q_{d}+\frac{132}{5} B q_{s}+\frac{1}{\lambda^{\prime}}\left(\frac{6}{5} B q_{d}-\frac{36}{5} B q_{s}\right)^{2}\right)
$$


These two equations are used to compute the Flumerfelt results cited in $\S 4$. In the special case of a clean droplet, the first-order expansion in $\mathrm{Ca}$ reduces to Taylor's equations (3.1) and (3.2) and reduces to Cox's equations (3.3) in any cases. Note that here, the inclination angle is measured between flow direction and the longest axis of the droplet which explains the sign - in the right member after $\pi / 4$. Flumerfelt (1980) defines the angle between the normal to the flow direction and the longest axis.

\section{REFERENCES}

Abreu, D., Levant, M., Steinberg, V. \& Seifert, U. 2014 Fluid vesicles in flow. Adv. Colloid Interface Sci. 208, 129-141.

Acrivos, A. 1983 The breakup of small drops and bubbles in shear flows. Ann. N.Y. Acad. Sci. 404, $1-11$.

BARThÈs-BIESEL, D. 2009 Capsule motion in flow: deformation and membrane buckling. $C$. $R$. Phys. 10 (8), 764-774.

BARTHÈs-BIESEl, D. \& ACRivos, A. 1973 Deformation and burst of a liquid droplet freely suspended in a linear shear field. J. Fluid Mech. 61 (01), 1-22.

BARTHÈs-BIESEL, D. \& SGAIER, H. 1985 Role of membrane viscosity in the orientation and deformation of a spherical capsule suspended in shear flow. J. Fluid Mech. 160, 119-135.

Boedec, G., Jaeger, M. \& Leonetti, M. 2012 Settling of a vesicle in the limit of quasispherical shapes. J. Fluid Mech. 690, 227-261.

Boedec, G., Jaeger, M. \& Leonetti, M. 2014 Pearling instability of a cylindrical vesicle. J. Fluid Mech. 743, 262-279.

Boedec, G., Leonetti, M. \& JAeger, M. 2011 3D vesicle dynamics simulations with a linearly triangulated surface. J. Comput. Phys. 230 (4), 1020-1034.

BoussinesQ, M. 1913 Sur l'existence d'une viscosité superficielle, dans la mince couche de transition séparant un liquide d'un autre fluide contigue. Ann. Chim. Phys. 29, 349-357.

Chaffey, C. E. \& BRenner, H. 1967 A second-order theory for shear deformation of drops. J. Colloid Interface Sci. 24, 258-269.

ChANG, K. \& Olbricht, W. 1993 Experimental studies of the deformation and breakup of a synthetic capsule in steady and unsteady simple shear flow. J. Fluid Mech. 250 (1), 609-633.

Cirak, F., Ortiz, M. \& SChroder, P. 2000 Subdivision surfaces: a new paradigm for thin-shell finite-element analysis. Intl J. Numer. Meth. Engng 47 (12), 2039-2072.

Cox, R. 1969 The deformation of a drop in a general time-dependent fluid flow. J. Fluid Mech. 37 (03), 601-623.

Cristini, V., Guido, S., Alfani, A., BŁawzdziewicz, J.\& Loewenberg, M. 2003 Drop breakup and fragment size distribution in shear flow. J. Rheol. 47 (5), 1283-1298.

Danov, K. D. 2001 On the viscosity of dilute emulsions. J. Colloid Interface Sci. 235 (1), 144-149.

Dickinson, E., Murray, B. S. \& Stainsby, G. 1988 Coalescence stability of emulsion-sized droplets at a planar oil-water interface and the relationship to protein film surface rheology. J. Chem. Soc. Faraday Trans. 84 (3), 871-883.

ERNi, P. 2011 Deformation modes of complex fluid interfaces. Soft Matt. 7 (17), 7586-7600.

ERni, P., Fischer, P. \& Windhab, E. J. 2005 Deformation of single emulsion drops covered with a viscoelastic adsorbed protein layer in simple shear flow. Appl. Phys. Lett. 87 (24), 244104.

Feigl, K., Megias-Alguacil, D., Fischer, P. \& Windhab, E. J. 2007 Simulation and experiments of droplet deformation and orientation in simple shear flow with surfactants. Chem. Engng Sci. 62 (12), 3242-3258.

Fischer, P. \& ERNI, P. 2007 Emulsion drops in external flow fields - the role of liquid interfaces. Curr. Opin. Colloid Interface Sci. 12 (4), 196-205.

Flumerfelt, R. W. 1980 Effects of dynamic interfacial properties on drop deformation and orientation in shear and extensional flow fields. J. Colloid Interface Sci. 76 (2), 330-349. 
Georgieva, D., Schmitt, V., Leal-Calderon, F. \& Langevin, D. 2009 On the possible role of surface elasticity in emulsion stability. Langmuir 25 (10), 5565-5573.

Guido, S., Greco, F. \& Villone, M. 1999 Experimental determination of drop shape in slow steady shear flow. J. Colloid Interface Sci. 219, 298-309.

Guido, S. \& Villone, M. 1998 Three-dimensional shape of a drop under simple shear flow. J. Rheol. 42, 395-415.

Harvey, P., Nguyen, A., Jameson, G. \& Evans, G. 2005 Influence of sodium dodecyl sulphate and dowfroth frothers on froth stability. Miner. Engng 18 (3), 311-315.

Huang, W.-X., Chang, C. B. \& Sung, H. J. 2012 Three-dimensional simulation of elastic capsules in shear flow by the penalty immersed boundary method. J. Comput. Phys. 231, 3340-3364.

Kennedy, M., Pozrikidis, C. \& SkAlaK, R. 1994 Motion and deformation of liquid drops, and the rheology of dilute emulsions in simple shear flow. Comput. Fluids 23 (2), 251-278.

KWAK, S. \& PoZRIKIDIS, C. 1998 Adaptive triangulation of evolving, closed, or open surfaces by the advancing-front method. J. Comput. Phys. 145 (1), 61-88.

LAngevin, D. 2000 Influence of interfacial rheology on foam and emulsion properties. Adv. Colloid Interface Sci. 88 (1), 209-222.

LE, D. V. 2010 Effect of bending stiffness on the deformation of liquid capsules enclosed by thin shells in shear flow. Phys. Rev. E 82, 016318.

LeVan, M. D. 1981 Motion of a droplet with a Newtonian interface. J. Colloid Interface Sci. 83 (1), 11-17.

Li, J., RenARDY, Y. Y. \& RENARdY, M. 2000 Numerical simulation of breakup of a viscous drop in simple shear flow through a volume-of-fluid method. Phys. Fluids 12 (2), 269-282.

LI, X. \& PozRikidis, C. 1997 The effect of surfactants on drop deformation and on the rheology of dilute emulsions in Stokes flow. J. Fluid Mech. 341, 165-194.

Loop, C. T. 1987 Smooth subdivision surfaces based on triangles. Master's thesis, University of Utah.

De Loubens, C., Deschamps, J., Boedec, G. \& Leonetti, M. $2015 a$ Stretching of capsules in an elongation flow, a route to constitutive law. J. Fluid Mech. 767, R3.

de Loubens, C., Deschamps, J., Edwards-Levy, F. \& Leonetti, M. $2015 b$ Tank-treading of microcapsules in shear flow. J. Fluid Mech., doi:10.1017/jfm.2015.758.

MAnor, O., LAVRenteVA, O. \& NIR, A. 2008 Effect of non-homogeneous surface viscosity on the marangoni migration of a droplet in viscous fluid. J. Colloid Interface Sci. 321 (1), 142-153.

Miller, R., Ferri, J. K., Javadi, A., Kragel, J., Mucic, N. \& Wustneck, R. 2010 Rheology of interfacial layers. Colloid Polym. Sci. 288, 937-950.

Mun, S. \& MCClements, D. J. 2006 Influence of interfacial characteristics on Ostwald ripening in hydrocarbon oil-in-water emulsions. Langmuir 22 (4), 1551-1554.

Narsimhan, V., Spann, A. P. \& Shaqfeh, E. S. G. 2015 Pearling, wrinkling buckling of vesicles in elongation flows. J. Fluid Mech. 777, 1-26.

OLDROYD, J. 1955 The effect of interfacial stabilizing films on the elastic and viscous properties of emulsions. Proc. R. Soc. Lond. A 232 (1191), 567-577.

Pawar, Y. \& STEBE, K. J. 1996 Marangoni effects on drop deformation in an extensional flow: the role of surfactnt physical chemistry. i. Insoluble surfactants. Phys. Fluids 8 (7), 1738-1751.

Phillips, W. J., Graves, R. W. \& Flumerfelt, R. W. 1980 Experimental studies of drop dynamics in shear fields: role of dynamic interfacial effects. J. Colloid Interface Sci. 76 (2), 350-370.

PozRIKIDIS, C. 1992 Boundary Integral and Singularity Methods for Linearized Viscous Flow. Cambridge University Press.

POZRIKIDIS, C. 1994 Effects of surface viscosity on the finite deformation of a liquid drop and the rheology of dilute emulsions in simple shearing flow. J. Non-Newtonian Fluid Mech. 51 (2), $161-178$.

RALlison, J. 1980 Note on the time-dependent deformation of a viscous drop which is almost spherical. J. Fluid Mech. 98 (03), 625-633.

RALLISON, J. 1981 A numerical study of the deformation and burst of a viscous drop in general shear flows. J. Fluid Mech. 109, 465-482. 
RAllison, J. \& ACRivos, A. 1978 A numerical study of the deformation and burst of a viscous drop in an extensional flow. J. Fluid Mech. 89 (01), 191-200.

RALlison, J. M. 1984 The deformation of small viscous drops and bubbles in shear flows. Annu. Rev. Fluid Mech. 16, 45-66.

Reusken, A. \& Zhang, Y. 2013 Numerical simulation of incompressible two-phase flows with a Boussinesq-Scriven interface stress tensor. Intl J. Numer. Meth. Fluids 73 (12), 1042-1058.

Rodrigues, D. S., Ausas, R. F., Mut, F. \& Buscaglia, G. C. 2015 A semi-implicit finite element method for viscous lipid membranes. J. Comput. Phys. 298, 565-584.

RumscheidT, F.-D. \& Mason, S. 1961 Particle motions in sheared suspensions XII. Deformation and burst of fluid drops in shear and hyperbolic flow. J. Colloid Sci. 16 (3), 238-261.

Schwalbe, J. T., Phelan, F. R. JR, Vlahovska, P. M. \& Hudson, S. D. 2011 Interfacial effects on droplet dynamics in Poiseuille flow. Soft Matt. 7 (17), 7797-7804.

SCRIVEN, L. 1960 Dynamics of a fluid interface equation of motion for Newtonian surface fluids. Chem. Engng Sci. 12 (2), 98-108.

Secomb, T. \& Skalak, R. 1982 Surface flow of viscoelastic membranes in viscous fluids. $Q$. $J$. Mech. Appl. Maths 35 (2), 233-247.

Spann, A. P., ZhaO, H. \& Shaqfeh, E. S. G. 2014 Loop subdivision surface boundary integral method simulations of vesicles at low reduced volume ratio in shear and extensional flow. Phys. Fluids 26, 031902.

Stone, H. \& Leal, L. 1990 The effects of surfactants on drop deformation and breakup. J. Fluid Mech. 220, 161-186.

Stone, H. A. 1994 Dynamics of drop deformation and breakup in viscous fluids. Annu. Rev. Fluid Mech. 26 (1), 65-102.

TAYlor, G. 1934 The formation of emulsions in definable fields of flow. Proc. R. Soc. Lond. A 146 (858), 501-523.

Valkovska, D., Danov, K. \& IVAnov, I. 1999 Surfactants role on the deformation of colliding small bubbles. Colloids Surf. A 156 (1), 547-566.

Vlahovska, P. M., Blawzdziewicz, J. \& Loewenberg, M. 2005 Deformation of a surfactantcovered drop in a linear flow. Phys. Fluids 17, 103103.

Vlahovska, P. M., Blawzdziewicz, J. \& Loewenberg, M. 2009a Small-deformation theory for a surfactant drop in linear flows. J. Fluid Mech. 624, 293-337.

Vlahovska, P. M., Podgorski, T. \& Misbah, C. $2009 \mathrm{~b}$ Vesicles and red blood cells in flow: from individual dynamics to rheology. C. R. Phys. 10 (8), 775-789.

YAZDANI, A. \& BAGCHI, P. 2013 Influence of membrane viscosity on capsule dynamics in shear flow. J. Fluid Mech. 718, 569-595.

Zell, Z. A., Nowbahar, A., Mansard, V., Leal, L. G., Deshmukh, S. S., Mecca, J. M., Tucker, C. J. \& SQuires, T. M. 2014 Surface shear inviscidity of soluble surfactants. Proc. Natl Acad. Sci. USA 111 (10), 3677-3682. 